\title{
A new species of Homo from the Late Pleistocene of the Philippines
}

Florent Détroit ${ }^{1 *}$, Armand Salvador Mijares ${ }^{2,3 *}$, Julien Corny ${ }^{1}$, Guillaume Daver ${ }^{4}$, Clément Zanolli $^{5,6}$, Eusebio Dizon ${ }^{3}$, Emil Robles ${ }^{2}$, Rainer Grün ${ }^{7,8}$ \& Philip J. Piper ${ }^{3,9}$

${ }^{1}$ Département Homme \& Environnement, Muséum National d'Histoire Naturelle, UMR 7194, CNRS, Musée de l'Homme, Paris, France.

${ }^{2}$ Archaeological Studies Program, University of the Philippines, Quezon City, The Philippines.

${ }^{3}$ National Museum of the Philippines, Manila, The Philippines.

${ }^{4}$ Laboratoire Paléontologie Evolution Paléoécosystèmes Paléoprimatologie (PALEVOPRIM), UMR 7262, CNRS, Université de Poitiers, Poitiers, France.

${ }^{5}$ Laboratoire PACEA, UMR 5199 CNRS, Université de Bordeaux, Bordeaux, France.

${ }^{6}$ Laboratoire AMIS, UMR 5288 CNRS, Université Toulouse III Paul Sabatier, Toulouse, France.

${ }^{7}$ Australian Research Centre for Human Evolution, Environmental Futures Research Institute, Griffith University, Brisbane, Queensland, Australia.

${ }^{8}$ Research School of Earth Sciences, Australian National University, Canberra, Australian Capital Territory, Australia.

${ }^{9}$ School of Archaeology and Anthropology, Australian National University,Canberra, Australian Capital Territory, Australia.

*e-mail: florent.detroit@mnhn.fr; mandy24_us@yahoo.com

\begin{abstract}
A hominin third metatarsal discovered in 2007 in Callao Cave (Northern Luzon, the Philippines) and dated to 67 thousand years ago provided the earliest direct evidence of a human presence in the Philippines. Analysis of this foot bone suggested that it belonged to the genus Homo, but to which species was unclear. Here we report the discovery of twelve additional hominin elements that represent at least three individuals that were found in the same stratigraphic layer of Callao Cave as the previously discovered metatarsal. These specimens display a combination of primitive and derived morphological features that is different from the combination of features found in other species in the genus Homo (including Homo floresiensis and Homo sapiens) and warrants their attribution to a new species, which we name Homo luzonensis. The presence of another and previously unknown hominin species east of the Wallace Line during the Late Pleistocene epoch underscores the importance of island Southeast Asia in the evolution of the genus Homo.
\end{abstract}


Continued excavations in Callao Cave (Fig. 1) that originally yielded the hominin third metatarsal1,2 (which we here call CCH1 for 'Callao Cave Hominin 1') have produced another twelve hominin elements (Extended Data Fig. 1a) from the same stratigraphic layer (layer 14): seven postcanine maxillary teeth (CCH6-a to CCH6-e, $\mathrm{CCH} 8, \mathrm{CCH} 9$; Fig. 2a, f, g), two manual phalanges ( $\mathrm{CCH} 2$ and $\mathrm{CCH} 5$; Fig. 2b, c), two pedal phalanges (CCH3 and $\mathrm{CCH} 4$; Fig. 2d, e) and a femoral shaft (CCH7; Fig. 2h). $\mathrm{CCH} 1$ and $\mathrm{CCH} 6-\mathrm{a}$ are directly dated by U-series analysis to minimum ages of 67 thousand years (kyr)1 and 50 kyr3, respectively. Crown morphology, grade of occlusal wear and exact correspondences of interproximal contact facets demonstrate that five of the upper right teeth belonged to a single individual (CCH6-a to CCH6-e; Extended Data Fig. $1 \mathrm{~b}-\mathrm{g}$ and Supplementary Information). The presence of two right upper third molars (M3; CCH6a and $\mathrm{CCH} 9)$ and a juvenile femoral shaft $(\mathrm{CCH} 7)$ indicates that at least three individuals are represented. On the basis of the unique mosaic of primitive (that is, Australopithecus-like) and derived (that is, H. sapiens-like) morphological features observed on these specimens, we assign them to a new species, $H$. luzonensis.

Order Primates Linnaeus, 1758

Suborder Anthropoidea Mivart, 1864

Superfamily Hominoidea Gray, 1825

Family Hominidae Gray, 1825

Tribe Hominini Gray, 1825

Genus Homo Linnaeus, 1758

Homo luzonensis sp. nov.

Etymology. The species name is derived from the island of Luzon, where the specimens were discovered.

Holotype. CCH6 (CCH6-a to CCH6-e), maxillary right postcanine dentition of a single individual discovered on 24 August 2011. The repository is the National Museum of the Philippines, Manila. Homo luzonensis has been deposited in the ZooBank database (http://zoobank.org/) with Life Science Identifier urn:lsid:zoobank. org:act:4F743862-662F-4E6B-9812-8A05533C1347.

Paratypes. Recovered in 2007, 2011 and 2015 from the same excavation area and layer as the holotype: $\mathrm{CCH} 1$, a right third metatarsal1; $\mathrm{CCH} 2$ and $\mathrm{CCH} 5$, two manual phalanges; $\mathrm{CCH} 3$ and $\mathrm{CCH} 4$, two pedal phalanges; $\mathrm{CCH} 8$, a left upper third or fourth premolar (P3/4); and $\mathrm{CCH}$, a right M3 (all specimens are housed at the National Museum of the Philippines, Manila).

Referred material. $\mathrm{CCH}$, a femoral shaft that belonged to a juvenile individual (housed at the National Museum of the Philippines, Manila).

Locality. The type locality is Callao Cave, in the Callao Limestone formation in the Peñablanca region of northern Luzon, the Philippines, at coordinates $17^{\circ} 42^{\prime} 11.7^{\prime \prime} \mathrm{N}, 121^{\circ} 49^{\prime} 25.5^{\prime \prime} \mathrm{E}$. 
Diagnosis. Postcanine maxillary teeth of small size that are mesiodistally compressed, with a premolar:molar crown size ratio that is high compared to other species in the genus Homo. Upper premolars with two or three roots, a mesio-distally expanded lingual crown, strong buccal grooves, partial or continuous transverse crest, and an enamel- dentine junction (EDJ) shape that is distinct from that of $H$. sapiens, Homo neanderthalensis and Asian Homo erectus. Very small upper molars, with a M1 > M2 > M3 crown size pattern, a simplified occlusal morphology with reduced metacone and hypocone, no crenulation on the EDJ, and EDJ shape affinities with that of $H$. sapiens and Asian $H$. erectus. Intermediate manual phalanx (rays 2-4) that is long and narrow (unlike all hominins except $H$. sapiens), with a longitudinally curved and dorso-palmarly compressed shaft, well-developed flexor sheath attachments and a strongly developed dorsal beak; it shares shape affinities with Australopithecus, $H$. floresiensis and - to a lesser extent- $H$. sapiens. Distal hand phalanx with proportions unlike $H$. neanderthalensis. The third metatarsal has a base that is very small relative to bone length, dorso-plantarly short and with a marked dorsoplantar convexity of the proximal articular facet. The proximal pedal phalanx (rays 2-4) shares shape affinities with Australopithecus: marked longitudinal curvature, parallel medial and lateral sides in dorsal view, a small bicondylar head and weak dorsal canting of the proximal articular surface (unlike African and European early Homo, H. naledi, H. neanderthalensis, H. floresiensis and H. sapiens).

\section{Description and comparison of the fossil elements}

Of the seven posterior maxillary teeth, six are from the right side (CCH6-a to CCH6-e and CCH9) and one (CCH8) is from the left (Fig. 2a, f, g, Extended Data Fig. 1 and Supplementary Information). The M2 (CCH6-b) and one of the M3s (CCH9) are complete; the other teeth have well-preserved crowns, and at least one-but sometimes all-roots are partly broken. Microfissures are present in the enamel, dentine and cementum, and the pulp cavity is generally filled by calcium carbonate; however, these post-depositional modifications do not affect the size, shape or morphological attributes of the teeth. In comparison to Australopithecus, Paranthropus and other species of the genus Homo, the maxillary postcanine teeth of H. luzonensis are small and mesiodistally compressed, and there is a marked contrast between the relative size and shape of the premolars and molars (Figs. 2-4 and Extended Data Figs. 1-4). The upper molars of H. luzonensis have small crown sizes with a M1 > M2 > M3 pattern and simplified occlusal surface morphology with a reduced number of cusps and an absence of marked crenulations at the EDJ (Fig. 3 and Extended Data Fig. 1). These derived features are found in other Late Pleistocene species of the genus Homo (that is, $H$. neanderthalensis, $H$. floresiensis and $H$. sapiens), but in this respect $H$. luzonensis molars more closely resemble those of $H$. sapiens4,5. However, the M1 and M2 of $H$. luzonensis have smaller crown sizes than those of H. sapiens (Fig. 3 and Extended Data Fig. 2d, e). Compared to the molars of H. floresiensis, H. luzonensis molars are smaller and the M1 is not more mesio-distally compressed than the other postcanine teeth6,7 (Extended Data Figs. 2-4). For their diminutive size and simplified crown morphology, $H$. luzonensis molars also differ considerably from Asian H. erectus4,5,8,9 and Denisovans10, although the EDJ of $H$. luzonensis M1 shows some shape affinities with several Indonesian $H$. erectus specimens (Extended Data Fig. 4f, h). H. luzonensis premolars are noticeably large relative to the molars - even more so than seen in H. floresiensis and unlike the pattern seen in other hominins, except Paranthropus (Fig. 3)- 
and also display several primitive features. For instance, the P3 and P4 crowns are asymmetric with a large and mesially displaced lingual cusp, as in early Homo11, and complete or partial mesial transverse ridges are present on the upper premolars, similar to those of $H$. floresiensis6,7. Developed mesial and distal vertical grooves are also evident on the buccal aspects of the P3 and P4; features that are more frequent in early Homo than in H. sapiens 12 and that are absent in $H$. floresiensis6,7. Moreover, H. luzonensis premolars have multiple roots (P3 has three, P4 has two) that are robust and highly divergent, an archaic condition typically found in Australopithecus, Paranthropus and early Homo13. Multiple robust and divergent premolar roots also occur in Asian H. erectus (Early Pleistocene Indonesian and Middle Pleistocene Chinese specimens), but are rare in H. sapiens and absent in H. floresiensis4,6,9,12-17. CCH8 (Fig. 2f and Extended Data Fig. 1f), an isolated left upper premolar, exhibits three roots (typically a P3 feature) with a relatively symmetric crown (typically a $\mathrm{P} 4$ feature). If $\mathrm{CCH} 8$ is a $\mathrm{P} 4$, a similar three-rooted condition has not been reported for any Pleistocene Homo from Asia and is rarely found in modern humans ( 1 to $3 \%) 13,14$. In terms of absolute tooth size and premolar-molar proportions, $H$. luzonensis shows a pattern that is not seen elsewhere in the genus Homo (Fig. 3 and Extended Data Fig. 2). Although Paranthropus shows similarly large premolars relative to molars (Fig. 3), the maxillary postcanine teeth of H. luzonensis differ markedly from those of Paranthropus in absolute size and shape (Extended Data Fig. 2). Multivariate statistical analysis of P3-M2 dental diameters results in $H$. luzonensis clustering with Asian $H$. erectus. Both species are characterized by almost similarly sized first and second molars, large premolars relative to molars and postcanine teeth that are mesio-distally compressed (Extended Data Fig. 2g, h). The mesio-distally compressed shape of the M1 of $H$. luzonensis is also visible in the results of the elliptic Fourier analysis of the crown contour, in which $H$. luzonensis plots at the margins of the range of variation for $H$. sapiens (which is characterized by more squared or rhomboidal M1s) (Extended Data Fig. 3). H. luzonensis differs from $H$. floresiensis, which displays an M1 crown contour shape that is even more mesio-distally compressed and has a more developed protocone (Extended Data Fig. 3c, f). Threedimensional geometric morphometric analyses of the P3 and P4 EDJs discriminate CCH6 and CCH8 from fossil and extant $H$. sapiens, $H$. neanderthalensis and Asian $H$. erectus, with $H$. luzonensis occupying its own area of the morphospace or sharing it with H. floresiensis when the latter is included (Fig. 4 and Extended Data Fig. 4d, e). The 3D geometric morphometric analyses of M1 and M2 EDJs are less clear, with $H$. luzonensis clustering with extant $H$. sapiens and Asian $H$. erectus (Extended Data Fig. 4f), with extant H. sapiens only (Extended Data Fig. 4g), with Asian H. erectus only (Extended Data Fig. 4h), or by itself (Extended Data Fig. 4i). However, the overall pattern of morphology seen in the dental remains of $H$. luzonensis is easily distinguishable from all previously described hominin species. $\mathrm{CCH} 2$ is an intermediate manual phalanx with a total length of 32.5 mm (interarticular length, $31 \mathrm{~mm}$; Fig. 2b, Supplementary Information and Supplementary Table 2 ). The bone is complete and well-preserved, but it was recovered broken just distal of the midshaft in two refitting parts (Extended Data Fig. 5a). On the basis of the asymmetry of the proximal and distal ends, it belongs to rays 2, 3 or 4 of the left hand. $\mathrm{CCH} 2$ displays primitive features such as an elongated and dorso-palmarly compressed shaft and well-developed flexor sheath attachments (Extended Data Fig. 5b). All these features are observed in Australopithecus 18-20 and to a lesser extent in the Homo habilis holotype OH721 (but see a previous publication22), but not in other Homo species that show more derived morphologies23-26 (Extended Data Fig. 5c), although a 
primitive morphology is reported for H. naledi27. Metric (Extended Data Fig. 5d-f) and 3D geometric morphometric analyses (Extended Data Fig. 6) show that the intermediate manual phalanx of $H$. luzonensis is long, narrow medio-laterally (base, shaft and head) and markedly curved. These shape characteristics are shared with Australopithecus and $H$. floresiensis, but not with $H$. naledi (which shares shape affinities with Paranthropus/early Homo specimens from Swartkrans), and are seen occasionally in $\mathrm{H}$. sapiens (Extended Data Fig. 6a, d, e). H. floresiensis differs from H. luzonensis and most other hominins by the shape of the phalangeal head, whichin lateral or medial view - has a very small diameter and is only slightly palmarly deviated relative to the proximo-distal axis of the shaft (Extended Data Fig. 6b, c). An idiosyncratic feature that differentiates $\mathrm{H}$. luzonensis from all other species of Homo is the strong development and proximal projection of the dorsal beak and this may have limited extension at the interphalangeal joint. CCH5 is a complete and well-preserved distal manual phalanx $15.9 \mathrm{~mm}$ in total length (interarticular length, 15.6 mm; Fig. 2c, Extended Data Fig. 7, Supplementary Information and Supplementary Table 2). Its laterality and ray cannot be determined with certainty, but its proximal articular surface is too small to articulate with $\mathrm{CCH} 2$. The proportions of $\mathrm{CCH} 5$ (apical tuft expansion and robusticity indices) are within the ranges of variation for H. sapiens and Australopithecus and outside those of H. neanderthalensis and H. floresiensis23,28 (Extended Data Fig. 7d, e). CCH4 is a complete and well-preserved right proximal pedal phalanx of rays 2, 3 or 4 (Fig. 2d, Extended Data Fig. 8, Supplementary Information and Supplementary Table 2) that is $27.4 \mathrm{~mm}$ long (interarticular length, $26.5 \mathrm{~mm}$ ). It shows primitive-Australopithecus-like- features, including pronounced longitudinal curvature of the shaft in lateral view, parallel medial and lateral diaphyseal margins in dorsal view and well-developed flexor sheath attachments in the distal two-thirds of the shaft, bordered by marked plantar crests (Extended Data Fig. 8b, c). The base is small, with a circular proximal articular surface that displays dorsal canting and articular angles of $94.5^{\circ}$ and $89.6^{\circ}$, respectively. These angular values are outside of the ranges of variation for $H$. sapiens, $H$. neanderthalensis, $H$. naledi and $H$. floresiensis, but within those of Australopithecus afarensis and Australopithecus africanus20,29-31 (Extended Data Fig. 8f, g). The head is relatively small, bicondylar and proximo-dorsally to disto-plantarly compressed, with a plantar breadth that is larger than its dorsal one. All of these features are typically found in combination in Australopithecus 19,20,29,31. The morphology of the proximal pedal phalanges of $H$. floresiensis has also been described as Australopithecus-like in some respects (for example, absence of an hourglass shape)32,33; however, the combination of features found in $\mathrm{CCH} 4$ is essentially indistinguishable from the features of A. afarensis and A. africanus as shown by a 3D geometric morphometric shape analysis (Fig. 5). Although proximal pedal phalanges are missing from the Asian $H$. erectus fossil record, those known for African and European species (for example, $H$. naledi, Homo antecessor and $H$. neanderthalensis) suggest that the morphology of $H$. luzonensis is unique among the genus Homo26,31,34. $\mathrm{CCH} 3$ is a complete and well-preserved intermediate pedal phalanx with a total length of $16.3 \mathrm{~mm}$ (interarticular length, $14.9 \mathrm{~mm}$; Fig. 2e, Extended Data Fig. 9, Supplementary Information and Supplementary Table 2). Because the shape, size and morphology of this element is highly variable in $H$. sapiens and other hominins, little can be said at this time about its comparative morphology; however, it visually more closely resembles the intermediate pedal phalanges of $\mathrm{H}$. sapiens and $\mathrm{H}$. floresiensis than it does those of $\mathrm{A}$. afarensis (Extended Data Fig. 9c). The previously published third metatarsal $(\mathrm{CCH} 1) 1,2$ has a proximal base 
that is particularly small compared to the total length of the bone. It is further characterized by a pronounced triangular shape with a short dorso-plantar height and a medio-laterally expanded dorsal aspect, and a marked dorso-plantar convexity of the proximal articular facet for the lateral cuneiform. The latter feature, which is very uncommon in hominins, has been reported (since the description of $\mathrm{CCH} 1$ ) in a fourth metatarsal of Australopithecus sediba35. The referred specimen, $\mathrm{CCH} 7$, is a partial left femur from which both the proximal and distal ends are missing (Fig. 2h, Extended Data Fig. 10 and Supplementary Information). Transverse slices of micro-computed tomography (micro-CT) scans show a high density of Haversian canals in the cortical bone that is characteristic of a growing juvenile individual (Extended Data Fig. 10c). Implications of $\boldsymbol{H}$. luzonensis for hominin evolution The Late Pleistocene hominin fossils from Callao Cave show a combination of dental and postcranial (hand and foot) features that is distinct from currently known species of the genus Homo (Supplementary Table 3). The premolars of H. luzonensis combine size and shape features seen in other Late Pleistocene species of the genus Homo (for example, $H$. neanderthalensis, $H$. floresiensis and $H$. sapiens) with primitive morphological features of the crown, EDJ and roots, which are typically found in earlier hominins, including Australopithecus and Paranthropus (Supplementary Table 3). The molars are extremely small and their external morphology resembles those of $H$. sapiens and-to a lesser extent-those of $H$. floresiensis, whereas some of their EDJ shapes show affinities with those of Asian H. erectus. Notable similarities are observed between H. luzonensis and Australopithecus in the anatomy of their manual and pedal elements. The morphologies of the hands and feet of Australopithecus, which are generally described as an intermediate between the morphologies of great apes and modern humans, are typically interpreted either as indicating adaptations to various degrees of bipedalism and climbing and/or suspension36 or as reflecting the retention of plesiomorphic features in obligatory bipeds35,37. However, the partial and fragmentary nature of the $H$. luzonensis postcranial elements presently limits further interpretation of its locomotor and manipulative abilities. The origin of $H$. luzonensis, as well as its phylogenetic relationships with other hominins present in eastern Asia at around the same time- including H. sapiens38, H. floresiensis39 and Denisovans10, and hominins recently discovered in China40-remains to be determined. As is the case for most hominins recovered from tropical Asia41, attempted DNA extraction from $H$. luzonensis fossils has been unsuccessful. Direct dates on H. luzonensis tooth and bone samples indicate it was present on Luzon before $50 \mathrm{kyr}$ ago1,3 and the recent discovery 42 of stone tools and a butchered rhinoceros in the nearby Cagayan Valley indicates that hominins, perhaps $H$. luzonensis, have been present on Luzon since more than $700 \mathrm{kyr}$ ago. Clearly, both H. luzonensis and $H$. floresiensis were present east of the Wallace Line on Luzon and Flores, respectively, at the same time39 and perhaps even over a similar temporal interval17,42. The skeletons of both species present anatomical traits that are either rare or absent elsewhere in the genus Homo but have similarities with those of Australopithecus6,7,23,32,33,43-45. As is the case with the island of Flores, a substantial sea crossing has always been required to reach Luzon from any mainland, even during the lowest sea level periods of the Quaternary period46 (Fig. 1). The insular nature of Luzon and the Philippine archipelago east of Palawan more generally is reflected in the high rates of endemism recognized in vertebrate faunas47-49. Given the highly endemic nature of the Luzon vertebrate faunas, evolutionary convergences and/or reversals under the effects of insular evolution50 may explain the distinct anatomy of H. luzonensis; however, further discoveries and 
more definitive evidence are needed. The discovery of $H$. luzonensis underscores the complexity of the evolution, dispersal and diversity of the genus Homo outside of Africa, and particularly in the islands of Southeast Asia, during the Pleistocene.

\section{References}

1. Mijares, A. S. et al. New evidence for a 67,000-year-old human presence at Callao Cave, Luzon, Philippines. J. Hum. Evol. 59, 123-132 (2010).

2. Détroit, F., Corny, J., Dizon, E. Z. \& Mijares, A. S. "Small size" in the Philippine human fossil record: is it meaningful for a better understanding of the evolutionary history of the Negritos? Hum. Biol. 85, 45-66 (2013).

3. Grün, R., Eggins, S., Kinsley, L., Moseley, H. \& Sambridge, M. Laser ablation U-series analysis of fossil bones and teeth. Palaeogeogr. Palaeoclimatol. Palaeoecol. 416, 150-167 (2014).

4. Kaifu, Y. et al. Taxonomic affinities and evolutionary history of the Early Pleistocene hominids of Java: dentognathic evidence. Am. J. Phys. Anthropol. 128, 709-726 (2005).

5. Zanolli, C. Additional evidence for morpho-dimensional tooth crown variation in a New Indonesian H. erectus sample from the Sangiran Dome (Central Java). PLoS ONE 8, e67233 (2013).

6. Kaifu, Y. et al. Descriptions of the dental remains of Homo floresiensis. Anthropol. Sci. 123, 129-145 (2015).

7. Kaifu, Y. et al. Unique dental morphology of Homo floresiensis and its evolutionary implications. PLoS ONE 10, e0141614 (2015).

8. Grine, F. E. \& Franzen, J. L. Fossil hominid teeth from the Sangiran dome (Java, Indonesia). Courier Forschungsinstitut Senckenberg (CFS) 171, 75-103 (1994).

9. Xing, S., Martinón-Torres, M. \& Bermúdez de Castro, J. M. The fossil teeth of the Peking Man. Sci. Rep. 8, 2066 (2018).

10. Sawyer, S. et al. Nuclear and mitochondrial DNA sequences from two Denisovan individuals. Proc. Natl Acad. Sci. USA 112, 15696-15700 (2015).

11. Gómez-Robles, A., Martinón-Torres, M., Bermúdez de Castro, J. M., Prado-Simón, L. \& Arsuaga, J. L. A geometric morphometric analysis of hominin upper premolars. Shape variation and morphological integration. J. Hum. Evol. 61, 688-702 (2011).

12. Xing, S. et al. Middle Pleistocene hominin teeth from Longtan Cave, Hexian, China. PLoS ONE 9, e114265 (2014).

13. Wood, B. A. \& Engleman, C. A. Analysis of the dental morphology of Plio-Pleistocene hominids. V. Maxillary postcanine tooth morphology. J. Anat. 161, 1-35 (1988).

14. Monsarrat, $P$. et al. Interrelationships in the variability of root canal anatomy among the permanent teeth: a full-mouth approach by cone-beam CT. PLOS ONE 11, e0165329 (2016).

15. Scott, G. R., Turner, C. G. II, Townsend, G. C. \& Martinón-Torres, M. The Anthropology of Modern Human Teeth: Dental Morphology and Its Variation in Recent and Fossil Homo sapiens (Cambridge Univ. Press, Cambridge, 2018).

16. Irish, J. D., Bailey, S. E., Guatelli-Steinberg, D., Delezene, L. K. \& Berger, L. R. Ancient teeth, phenetic affinities, and African hominins: another look at where Homo naledi fits in. J. Hum. Evol. 122, 108-123 (2018).

17. van den Bergh, G. D. et al. Homo floresiensis-like fossils from the early Middle Pleistocene of Flores. Nature 534, 245-248 (2016). 
18. Bush, M. E., Lovejoy, C. O., Johanson, D. C. \& Coppens, Y. Hominid carpal, metacarpal, and phalangeal bones recovered from the Hadar formation: 1974-1977 collections. Am. J. Phys. Anthropol. 57, 651-677 (1982).

19. Susman, R. L., Stern, J. T. Jr \& Jungers, W. L. Arboreality and bipedality in the Hadar hominids. Folia Primatol. 43, 113-156 (1984).

20. Ward, C. V., Kimbel, W. H., Harmon, E. H. \& Johanson, D. C. New postcranial fossils of Australopithecus afarensis from Hadar, Ethiopia (1990-2007). J. Hum. Evol. 63, 1-51 (2012).

21. Napier, J. Fossil hand bones from Olduvai Gorge. Nature 196, 409-411 (1962).

22. Moyà-Solà, S., Köhler, M., Alba, D. M. \& Almécija, S. Taxonomic attribution of the Olduvai hominid 7 manual remains and the functional interpretation of hand morphology in robust australopithecines. Folia Primatol. 79, 215-250 (2008).

23. Larson, S. G. et al. Descriptions of the upper limb skeleton of Homo floresiensis. J. Hum. Evol. 57, 555-570 (2009).

24. Trinkaus, E. The Shanidar Neandertals (Academic, London, 1983).

25. Walker, A. \& Leakey, R. E. The Nariokotome Homo erectus skeleton (Harvard Univ. Press, Cambridge, 1993).

26. Lorenzo, C., Arsuaga, J. L. \& Carretero, J. M. Hand and foot remains from the Gran Dolina early Pleistocene site (Sierra de Atapuerca, Spain). J. Hum. Evol. 37, 501-522 (1999).

27. Kivell, T. L. et al. The hand of Homo naledi. Nat. Commun. 6, 8431 (2015). 28. Mittra, E. S., Smith, H. F., Lemelin, P. \& Jungers, W. L. Comparative morphometrics of the primate apical tuft. Am. J. Phys. Anthropol. 134, 449-459 (2007).

29. Latimer, B. M., Lovejoy, C. O., Johanson, D. C. \& Coppens, Y. Hominid tarsal, metatarsal, and phalangeal bones recovered from the Hadar formation: 1974-1977 collections. Am. J. Phys. Anthropol. 57, 701-719 (1982).

30. Griffin, N. L. \& Richmond, B. G. Joint orientation and function in great ape and human proximal pedal phalanges. Am. J. Phys. Anthropol. 141, 116-123 (2010).

31. Trinkaus, E. \& Patel, B. A. An Early Pleistocene human pedal phalanx from Swartkrans, SKX 16699, and the antiquity of the human lateral forefoot. C. R. Palevol 15, 978-987 (2016).

32. Jungers, W. L. et al. Descriptions of the lower limb skeleton of Homo floresiensis. J. Hum. Evol. 57, 538-554 (2009).

33. Jungers, W. L. et al. The foot of Homo floresiensis. Nature 459, 81-84 (2009). 34. HarcourtSmith, W. E. H. et al. The foot of Homo naledi. Nat. Commun. 6, 8432 (2015).

35. DeSilva, J. M. et al. The lower limb and mechanics of walking in Australopithecus sediba. Science 340, 1232999 (2013).

36. Stern, J. T. J. Jr \& Susman, R. L. The locomotor anatomy of Australopithecus afarensis. Am. J. Phys. Anthropol. 60, 279-317 (1983).

37. Latimer, B., Ohman, J. C. \& Lovejoy, C. O. Talocrural joint in African hominoids: implications for Australopithecus afarensis. Am. J. Phys. Anthropol. 74, 155-175 (1987).

38. Westaway, K. E. et al. An early modern human presence in Sumatra 73,000-63,000 years ago. Nature 548, 322-325 (2017).

39. Sutikna, T. et al. Revised stratigraphy and chronology for Homo floresiensis at Liang Bua in Indonesia. Nature 532, 366-369 (2016).

40. Li, Z.-Y. et al. Late Pleistocene archaic human crania from Xuchang, China. Science 355, 969972 (2017).

41. Bellwood, P. The search for ancient DNA heads east. Science 361, 31-32 (2018).

42. Ingicco, T. et al. Earliest known hominin activity in the Philippines by 709 thousand years ago. Nature 557, 233-237 (2018). 
43. Brown, P. et al. A new small-bodied hominin from the Late Pleistocene of Flores, Indonesia. Nature 431, 1055-1061 (2004).

44. Jungers, W. L. in A Companion to Paleoanthropology (ed. Begun, D. R.) 582-598 (Blackwell, Hoboken, 2013).

45. Argue, D., Groves, C. P., Lee, M. S. Y. \& Jungers, W. L. The affinities of Homo floresiensis based on phylogenetic analyses of cranial, dental, and postcranial characters. J. Hum. Evol. 107, 107-133 (2017).

46. Voris, H. K. Maps of Pleistocene sea levels in Southeast Asia: shorelines, river systems and time durations. J. Biogeogr. 27, 1153-1167 (2000).

47. Heaney, L. R., Balete, D. S. \& Rickart, E. A. The Mammals of Luzon Island (Johns Hopkins Univ. Press, Baltimore, 2016).

48. Heaney, L. R. A synopsis of the mammalian fauna of the Philippine Islands. Fieldiana Zool. 88, 1-61 (1998).

49. van der Geer, A. A. E., Lomolino, M. V. \& Lyras, G. A. 'Island Life' before man: biogeography of palaeo-insular mammals. J. Biogeogr. 44, 995-1006 (2017).

50. van den Hoek Ostende, L. W. Cladistics and insular evolution, an unfortunate marriage? Another tangle in the Deinogalerix analysis of Borrani et al. (2017). Cladistics 34, 708-713 (2018).

Acknowledgements F.D., J.C., G.D. and C.Z. thank L. Puymerail. We thank T. Sutikna and W. Saptomo (Pusat Penelitian Arkeologi Nasional); X.-J. Wu and W. Liu (IVPP); J. de Vos (Naturalis); N. Giang Hai, N. Kim Thuy, N. Lan Cuong and N. Thi Mai Huong (Institute of Archaeology); N. Viet (Center for Southeast Asian Prehistory); A. Kijngam and R. Thosarat (Thai Fine Arts Department); the National Research Council of Thailand; G. Garcia-Pack, I. Tattersall and E. Delson (American Museum of Natural History); the Field Museum of Natural History; the Ethiopian Authority for Research and Conservation of the Cultural Heritage; the National Museums of Kenya and Government of Kenya; the Ditsong National Museum of Natural History, the Evolutionary Studies Institute, University of the Witwatersrand; the Centre de Valorisation des Collections scientifiques de l'Université de Poitiers; S. Bahuchet, A. Fort, M. Friess, A. Froment, D. Grimaud-Hervé, L. Huet, V. Laborde and P. Mennecier (MNHN); the Nespos Society (https://www.nespos.org); MorphoSource, Duke University (https://www.morphosource.org/); the Senckenberg Research Institute Frankfurt; the ESRF Paleontological Database (paleo.esrf.eu); P. Fernandez, C. Higham, W. Jungers, Y. Kaifu, R. Kono, H. Matsumura, M. Oxenham, N. Tayles and $\mathrm{M}$. Tocheri for granting access to or sharing information and data on recent and fossil hominins; M. Stoneking and Q. Fu (Max Planck Institute for Evolutionary Anthropology); AST-RX and Imagerie 2D/3D MH-SU platforms (MNHN); G. Champion, A. Giral, A. Gossez, A. Van Heteren, J. Surault and J. Tanedo for discussions or technical assistance; the National Museum of the Philippines and its Directorial staff; colleagues and students from the Archaeological Studies Program (University of the Philippines); the 2011 and 2015 IMQP Erasmus Mundus students; and the Cagayan Provincial Government and the Protected Area Management Board-Peñablanca for authorizing fieldwork at Callao Cave. Funding was provided by the Wenner- Gren Foundation, the Leakey Foundation Research Grant and the University of the Philippines Enhanced Creative Work and Research Grant (ECWRG 2015-1- 016). R.G.'s work was partly funded by the Australian Research Council Grant DP110101415. The research of P.J.P. was supported by Australian Research Council grants FT100100527 and DP140100384. The research of G.D. was supported by 
the Agence Nationale pour la Recherche (ANR ARCHOR- 12-CULT-0006), the Omo Group Research Expedition (J.-R. Boisserie), the MEAE, the Université de Poitiers and the Conseil départemental de la Vienne (ACI 2013-2014). The research of J.C. was supported by SYNTHESYS (NLTAF- 1130) and 'IUSS Ferrara 1391' grants. Aspects of the research of F.D. were supported by the Labex BCDiv 'Biological and Cultural Diversities', Erasmus Mundus IMQP and IDQP, MQPI (MEAE) and PaléoFED.

Author contributions F.D., J.C., G.D. and C.Z. analysed the hominin fossils, A.S.M. is the project leader, E.D. and E.R. are senior members of the excavation team, R.G. conducted the dating, F.D. and P.J.P. wrote the manuscript, F.D., J.C., G.D., C.Z. and P.J.P. edited the manuscript.

Competing interests The authors declare no competing interests. 


\section{Methods}

Computed tomography and virtual cleaning of the fossils. The H. luzonensis specimens were cleaned mechanically, following previously published procedures 1 . The thickest patches of sediment were removed, but the carbonated coating was usually left untouched to avoid damaging the underlying surface of the bones or teeth. Details on the state of preservation of each specimen are given in the Supplementary Information. Descriptions and comparisons mostly relied on observations made on the three-dimensional models that were reconstructed after virtual cleaning, which was performed during the segmentation of the micro-CT slices. The refitting of specimens broken in two parts $(\mathrm{CCH} 2$ and $\mathrm{CCH} 7)$ was also done virtually, after segmentation of the tomographic slices. The original fossil specimens were scanned at the AST-RX platform of the MNHN (Paris, France), using a GE Sensing \& Inspection Technologies phoenix $\mid X$-ray v|tome $\mid \mathrm{X}$ L240-180 CT scanner. The teeth (CCH6b-CCH6e and CCH8) were scanned with isotropic voxel sizes ranging from 0.0094 to $0.0143 \mathrm{~mm}$ (voltage $=80-90 \mathrm{kV}$; current $=120-350 \mu \mathrm{A}$ ). The bones $(\mathrm{CCH} 2, \mathrm{CCH} 3, \mathrm{CCH} 4, \mathrm{CCH} 5$ and $\mathrm{CCH} 7)$ were scanned with isotropic voxel sizes ranging from 0.0233 to $0.0647 \mathrm{~mm}$ (voltage $=80-100 \mathrm{kV}$; current $=300-350 \mu \mathrm{A})$. Semimanual segmentation using the half-maximum height protocol51 and three-dimensional modelling were performed in Avizo (Visualization Sciences Group). Photogrammetry. The two right M3s (CCH6-a and CCH9) were intentionally preserved from potential X-ray damage that may affect dating and ancient DNA analyses52-54; thus, three-dimensional surface models were obtained using photogrammetry (with Agisoft PhotoScan Professional and Geomagic Studio) at the 'imagerie 2D/3D' platform of Musée de l'Homme, MNHN. Measurements. The original fossil specimens were measured to the nearest $0.1 \mathrm{~mm}$ with a Mitutoyo sliding Vernier calliper (Supplementary Tables 1, 2). For premolars and molars, bucco-lingual and mesio-distal diameters were measured following previously published methods55, with correction of the mesio-distal diameter for interproximal wear. Dental crown measurements for specimens used in morphometric comparisons were averaged between right and left sides, when available. For the proximal pedal phalanx (CCH4), the dorsal canting angle of the proximal joint surface was measured following previously published studies30,56 and the articular angle following a previous publication31, adapted to a virtual midsagittal section. For the intermediate manual phalanx $(\mathrm{CCH} 2)$, the standard measurements of the maximum medio-lateral breadths of the base (MLbase), the midshaft (MLmid) and the head (MLend), as well as the interarticular length were measured following previously published methods 18 taken from a virtual midsagittal section. For the distal manual phalanx $\mathrm{CCH} 5$, the apical tuft maximum medio-lateral width, the maximum medio-lateral width of the base and the biomechanical length were measured following previously published methods 28 . Statistics and morphometric analyses. All statistical analyses and graphics were computed with $\mathrm{R}$ version 3.5.157, using several functions adapted from a previous study58 as well as the packages ade459, geomorph60, ggplot261, ggpubr62, momocs63, Morpho64, rg165, shapes66 and vegan67. For premolars and molars, uni- and bivariate analyses of dental metrics were made on raw measurements (bucco-lingual (BL) and mesio-distal (MD) diameters $)$ and on the square roots of computed occlusal surface areas $(\sqrt{ }(\mathrm{MD} \times \mathrm{BL}))$. The $\operatorname{bgPCAs}$ were computed on bucco-lingual and mesio-distal diameters transformed into log-shape ratios68. Among size-adjustment procedures, log-shape ratios are well-known for their statistical robustness and efficiency in distinguishing shape from absolute size differences69. CCH6 was considered to be a supplementary individual in the bgPCA and data were plotted a posteriori on bgPC1 and bgPC2. For intermediate manual phalanges, the robusticity index $(0.5 \times$ (midshaft breadth + midshaft height)/articular length $\times 100$ ) was calculated following previously published methods 26 and the medio-lateral base width index (MLbase $=$ medio-lateral width of the base/interarticular length $\times 100$ ), medio-lateral head width index (MLend $=$ mediolateral width of the 
head/interarticular length $\times 100$ ), and mediolateral midshaft width index (MLmid $=$ mediolateral width at midshaft/interarticular length $\times 100$ ) were calculated as previously described 70 . For distal manual phalanges, the robusticity and expansion indices were calculated according to previously published methods 28 (robusticity index $=$ apical tuft maximum medio-lateral width/biomechanical length $\times 100$; expansion index $=$ apical tuft maximum medio-lateral width/maximum mediolateral width of the base $\times 100)$. Shape affinities of the M1 (CCH6-c) were explored with elliptic Fourier analysis applied on Procrustes-aligned crown outlines, following previously published methodological and statistical procedures71,72. Comparative specimens included a large sample of archaeological $(n=105)$ and recent $(n=140) H$. sapiens from various southeast Asian populations, as well as $H$. floresiensis. Details on the samples are given in Supplementary Table 5 and in a previous study72. To thoroughly test the possible effects of the large and deep interproximal contact facet that is present on the mesial aspect of the right M1 of LB1, two different reconstructions were included in the analyses: the original crown contour that has previously been published7, and a slightly different reconstruction, with more compensation of the mesial interproximal contact facet (see Extended Data Fig. 3d). For premolars and molars, the virtual surfaces of the EDJ of the Callao specimens were compared with those of fossil and extant Homo specimens (Supplementary Table 6). The moderately worn dentine horn apices were reconstructed following a geometric method validated in a previous study73. To do so, the virtual slices of each tooth were resampled to be parallel to the cervical plane. This plane was then translated into each dentine horn extremity, and two sections perpendicular to the cervical plane-corresponding, respectively, to the widest mesio-distal and bucco-lingual diameters of the last section of the dentine horn and intersecting its centre-were used to reconstruct the height and orientation of each apex. Interpolations were then performed for rendering the three-dimensional shape of the tips. Landmarks were placed at the dentine horn apices of the paracone and protocone for the premolars, and of the paracone, protocone metacone and hypocone for the molars74. A set of sliding semilandmarks was positioned along the marginal ridges, as well as along the oblique crest on the molars74 (Extended Data Fig. 4a, b). To analyse the shape of the proximal pedal phalanx CCH4, it was compared with samples of Australopithecus, recent $H$. sapiens and $H$. floresiensis, restricted to proximal pedal phalanges of rays 2, 3 and 4 of the right foot (Australopithecus phalanges attributed to a left foot were mirrored), with no signs of pronounced osteoarthritis and/or osteophyte (details on the samples can be found in Supplementary Table 7). The only exception to the latter condition was the $H$. floresiensis proximal pedal phalanx LB1/38 - which presents a developed osteophyte on one side of its head-because "the value [of the curvature] seen in LB1/38 falls outside the human range and recalls the condition reported for some australopithecines" 32 . After the sliding of the semilandmarks (minimization of the bending energy criterion), we made sure that no semilandmarks were on the surface of the osteophyte of LB1/38. The analytical procedure involved 20 landmarks and 250 semilandmarks, equally spaced on the whole surface of the phalanges (Extended Data Fig. 8e). For intermediate manual phalanges, $\mathrm{CCH} 2$ was compared with samples of Australopithecus, Paranthropus/Homo, H. naledi, H. floresiensis and recent $H$. sapiens. For recent $H$. sapiens, the most complete individuals for intermediate manual phalanges were selected from the collections for the more accurate determinations of the rays, following a previously published method75. The siding of the phalanges was done using previously described techniques76,77. For each individual, intermediate phalanges of rays 2, 3 and 4 of the left hand were selected for analyses, but in case the right hand was more complete, right phalanges were mirrored and used instead (see details on the samples in Supplementary Table 9). The head of the H. floresiensis intermediate manual phalanx LB1/48, which is partly damaged on one side, was virtually reconstructed by mirroring the well-preserved side for this series of analyses. The 
analytical procedure involved 23 landmarks and 250 semilandmarks, equally spaced on the whole surface of the phalanges (Extended Data Fig. 6g). Generalized Procrustes analyses were performed with principal component analyses (PCAs) and bgPCAs based on the Procrustes shape coordinates 78 (Figs. 4 and 5 and Extended Data Figs. 4, 6). The analytical strategies followed for analysing the shape of proximal pedal phalanges and that of intermediate manual phalanges differ. For proximal pedal phalanges, all phalanges from rays 2, 3 and 4 were analysed together in a single Procrustes analysis, because inter-individual variation is greater than intra-individual (between rays) variation (that is, proximal pedal phalanges of rays 2, 3 and 4 of a single $H$. sapiens individual tend to cluster closely on PC1 versus PC2). By contrast, intra-individual variation is larger than inter-individual variation in recent $H$. sapiens when intermediate manual phalanges from rays 2,3 and 4 are analysed together (that is, the effect of the ray is dominant on PC1 versus PC2). Because the objective of this analysis was to test for the shape affinities of $H$. luzonensis in a taxonomical perspective, but not an attempt at determining the most probable ray to which $\mathrm{CCH} 2$ belonged, recent $H$. sapiens were subdivided into three distinct samples (one for each ray, from ray 2 to ray 4), which were analysed in three separate Procrustes analyses, each including all fossil specimens. In all sets of analyses (EDJ, proximal pedal phalanges and intermediate manual phalanges), the Callao specimens were included a posteriori in the bgPCAs. Comparative specimens. Australopithecus includes A. afarensis, A. africanus, Australopithecus anamensis and A. sediba; Paranthropus includes Paranthropus aethiopicus, Paranthropus boisei and Paranthropus robustus; African and European early Homo includes H. habilis, Homo rudolfensis, Homo ergaster, Homo georgicus and $H$. antecessor; Paranthropus/early Homo (phalanges only) includes specimens from Olduvai and Swartkrans whose generic attribution is debated (see main text and Extended Data Figs. 5-7), Asian early Homo includes H. erectus, H. cf. floresiensis (from Mata Menge, Flores, Indonesia); H. floresiensis includes the holotype LB1 and elements (phalanges) of LB6; $H$. sapiens includes recent specimens (including 'Negritos'), as well as Upper Pleistocene and Holocene specimens (except for EDJ analyses, for which fossil H. sapiens samples were distinguished from recent $H$. sapiens). For dental metrics, 'Negritos' were treated as a separate group in the analyses shown in Extended Data Fig. 2j-k. For pedal and manual phalanges, the three-dimensional models used in the geometric morphometrics analyses were obtained from scanning original specimens (recent H. sapiens) and high-quality casts (Australopithecus and Paranthropus/Homo) with Nextengine and Mechscan surface scanners. Three-dimensional models of $H$. floresiensis were provided by Pusat Penelitian Arkeologi Nasional, and those of A. sediba and $H$. naledi intermediate manual phalanges by the University of the Witwatersrand, Evolutionary Studies Institute through https://www.morphosource.org/, Duke University. Detailed lists of specimens included in each analysis (including analyses presented in Extended Data Figs. 2-8) and sources of data are given in Supplementary Tables 4-12.

Data availability The data that support the findings of this study are available from the corresponding authors upon reasonable request. Homo luzonensis has been deposited in the ZooBank database (http://zoobank.org/) with Life Science Identifier urn:lsid:zoobank. org:act:4F743862-662F-4E6B-9812-8A05533C1347. The description of the species has been deposited with Life Science identifier urn:lsid:zoobank.org:pub: 0E4607F1-1374-4842-B32B7CE2250807DF.

\section{References}


51. Spoor, C. F., Zonneveld, F. W. \& Macho, G. A. Linear measurements of cortical bone and dental enamel by computed tomography: applications and problems. Am. J. Phys. Anthropol. 91, 469-484 (1993).

52. Duval, M. \& Martín-Francés, L. Quantifying the impact of $\mu$ CT-scanning of human fossil teeth on ESR age results. Am. J. Phys. Anthropol. 163, 205-212 (2017).

53. Grün, R., Athreya, S., Raj, R. \& Patnaik, R. ESR response in tooth enamel to high-resolution CT scanning. Archaeol. Anthropol. Sci. 4, 25-28 (2012).

54. Immel, A. et al. Effect of X-ray irradiation on ancient DNA in sub-fossil bones - guidelines for safe X-ray imaging. Sci. Rep. 6, 32969 (2016).

55. Wood, B. Koobi Fora Research Project. Volume 4, Hominid Cranial Remains (Clarendon, Oxford, 1991).

56. Duncan, A. S., Kappelman, J. \& Shapiro, L. J. Metatarsophalangeal joint function and positional behavior in Australopithecus afarensis. Am. J. Phys. Anthropol. 93, 67-81 (1994).

57. R Core Team. R: A Language and Environment for Statistical Computing. (R Foundation for Statistical Computing, 2017).

58. Claude, J. Morphometrics with R (Springer, 2008).

59. Dray, S. \& Dufour, A.-B. The ade4 package: implementing the duality diagram for ecologists. J. Stat. Softw. 22, 1-20 (2007).

60. Adams, D. C., Collyer, M. L. \& Kaliontzopoulou, A. Geomorph: software for geometric morphometric analyses. R package version 3.0.6 https://cran.r-project.org/package=geomorph (2018).

61. Wickham, H. ggplot2: Elegant Graphics for Data Analysis (Springer-Verlag, 2009).

62. Kassambara, A. ggpubr: 'ggplot2' based publication ready plots. R package version 0.1.8 https://cran.r-project.org/web/packages/ggpubr/ (2017).

63. Bonhomme, V., Picq, S., Gaucherel, C. \& Claude, J. Momocs: outline analysis using R. J. Stat. Softw. 56, 1-24 (2014).

64. Schlager, S. in Statistical Shape and Deformation Analysis (eds Zheng, G. et al.) 217-256 (Academic, 2017).

65. Adler, D. et al. rgl: 3D visualization using OpenGL. R package version 0.99.16 https://cran.rproject.org/web/packages/rgl/ (2018).

66. Dryden, I. L. shapes. R package version 1.2.4 https://cran.r-project.org/web/packages/shapes/ (2017).

67. Oksanen, J. et al. vegan: community ecology package. R package version 2.5-4 https://cran.rproject.org/web/packages/vegan/ (2018).

68. Darroch, J. N. \& Mosimann, J. E. Canonical and principal components of shape. Biometrika 72, 241-252 (1985).

69. Jungers, W. L., Falsetti, A. B. \& Wall, C. E. Shape, relative size, and size adjustments in morphometrics. Am. J. Phys. Anthropol. 38, 137-161 (1995).

70. Musgrave, J. H. in Human Evolution Vol. 11 (ed. Day, M. H.) 59-85 (Taylor \& Francis, London, 1973).

71. Corny, J. \& Détroit, F. Anatomic identification of isolated modern human molars: testing procrustes aligned outlines as a standardization procedure for elliptic Fourier analysis. Am. J. Phys. Anthropol. 153, 314-322 (2014).

72. Corny, J. et al. Dental phenotypic shape variation supports a multiple dispersal model for anatomically modern humans in Southeast Asia. J. Hum. Evol. 112, 41-56 (2017). 
73. Zanolli, C. et al. The Middle Pleistocene (MIS 12) human dental remains from Fontana Ranuccio (Latium) and Visogliano (Friuli-Venezia Giulia), Italy. A comparative high resolution endostructural assessment. PLoS ONE 13, e0189773 (2018).

74. Zanolli, C. et al. Inner tooth morphology of Homo erectus from Zhoukoudian. New evidence from an old collection housed at Uppsala University, Sweden. J. Hum. Evol. 116, 1-13 (2018).

75. Susman, R. L. Comparative and functional morphology of hominoid fingers. Am. J. Phys. Anthropol. 50, 215-236 (1979).

76. Case, D. T. \& Heilman, J. New siding techniques for the manual phalanges: a blind test. Int. J. Osteoarchaeol. 16, 338-346 (2006).

77. Christensen, A. M. Techniques for siding manual phalanges. Forensic Sci. Int. 193, 84-87 (2009).

78. Mitteroecker, P. \& Bookstein, F. Linear discrimination, ordination, and the visualization of selection gradients in modern morphometrics. Evol. Biol. 38, 100-114 (2011). 
Figures

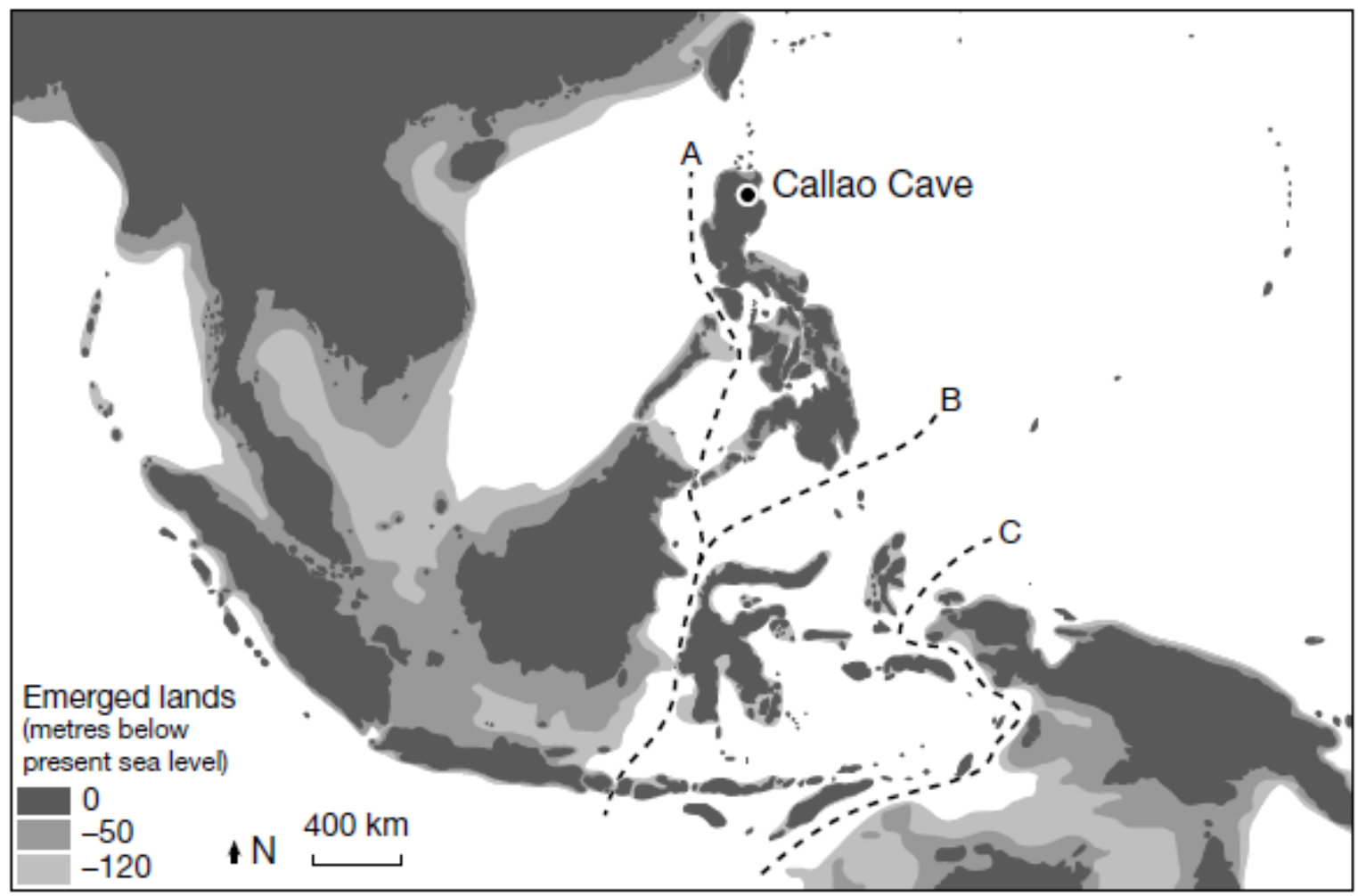

Fig. 1. Geographical location of Callao Cave. Map showing the location of Callao Cave on Luzon Island (the Philippines), emerged lands at 50 and $120 \mathrm{~m}$ below present sea level (adapted from ref. 46, H. K. Voris, Field Museum of Natural History) and the major biogeographical boundaries recognized in the area. A, Wallace's Line modified by Huxley; B, Wallace's Line; C, Lydekker's Line. Luzon Island lies in between the original Wallace's Line and the Wallace's Line modified by Huxley and was never connected to mainland Asia during the Quaternary. 

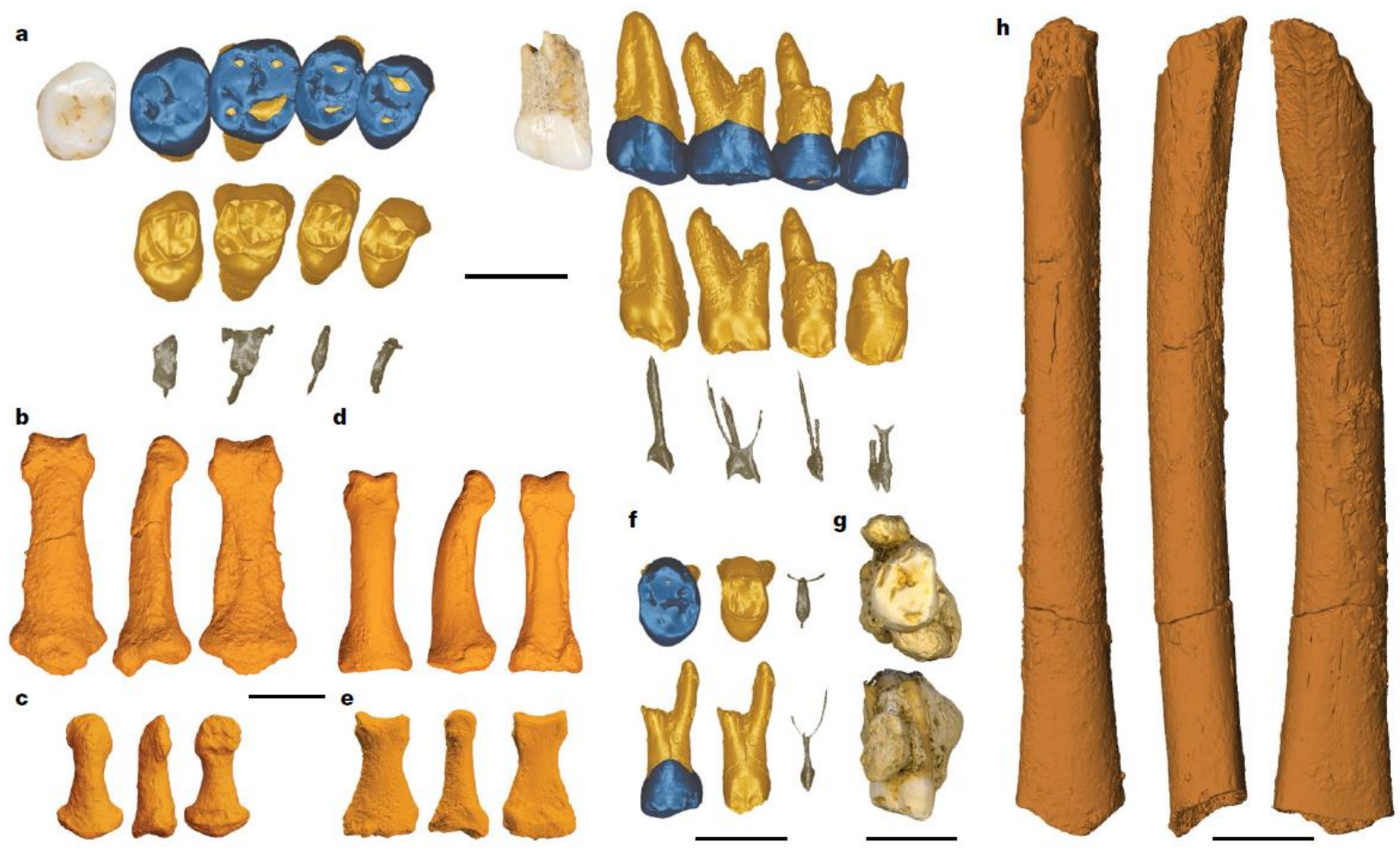

Fig. 2. Fossil remains of H. luzonensis from Late Pleistocene sediments at Callao Cave. a, Holotype CCH6: postcanine maxillary teeth in occlusal (left) and buccal (right) aspects, with three-dimensional rendering of enamel (dark blue), dentine and cement (light brown), and pulp cavity (dark grey) for CCH6-b-CCH6-e. b, Intermediate manual phalanx CCH2 (dorsal, lateral and palmar aspects). c, Distal manual phalanx CCH5 (dorsal, lateral/medial and palmar aspects). d, Proximal pedal phalanx CCH4 (dorsal, lateral and plantar aspects). e, Intermediate pedal phalanx $\mathrm{CCH} 3$ (dorsal, medial and plantar aspects). f, Left P3 or P4 CCH8: occlusal (top) and buccal (bottom) aspects, with three-dimensional rendering of enamel, dentine and cement, and pulp cavity. g, Right M3 CCH9: occlusal (top) and buccal (bottom) aspects. h, Juvenile femoral shaft CCH7 (anterior, lateral and posterior aspects). Scale bars, $10 \mathrm{~mm}(\mathrm{a}-\mathrm{g})$ and $20 \mathrm{~mm}(\mathrm{~h})$; additional views are shown in Extended Data Figs. 1, 5, 7-10. 
Australopithecus

Paranthropus

African and European early Homo

Asian early Homo

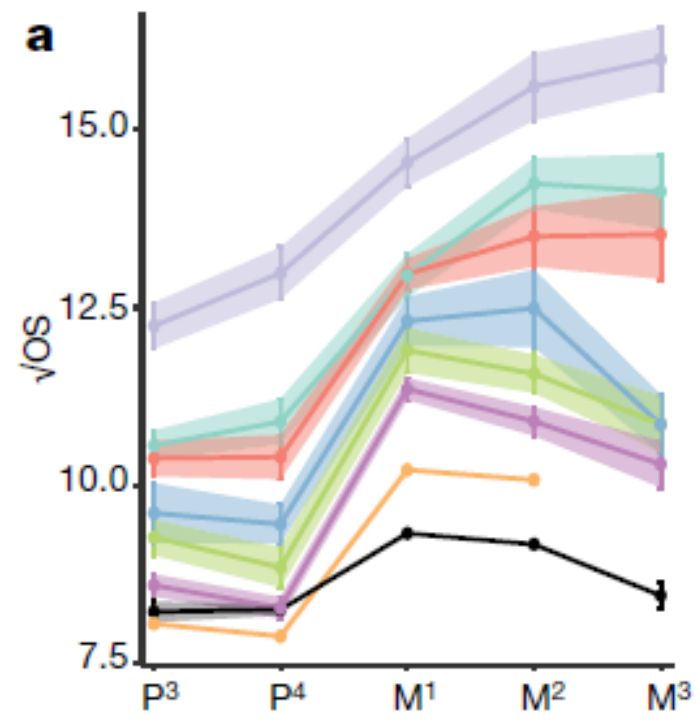

H. neanderthalensis

H. sapiens

H. floresiensis

H. luzonensis

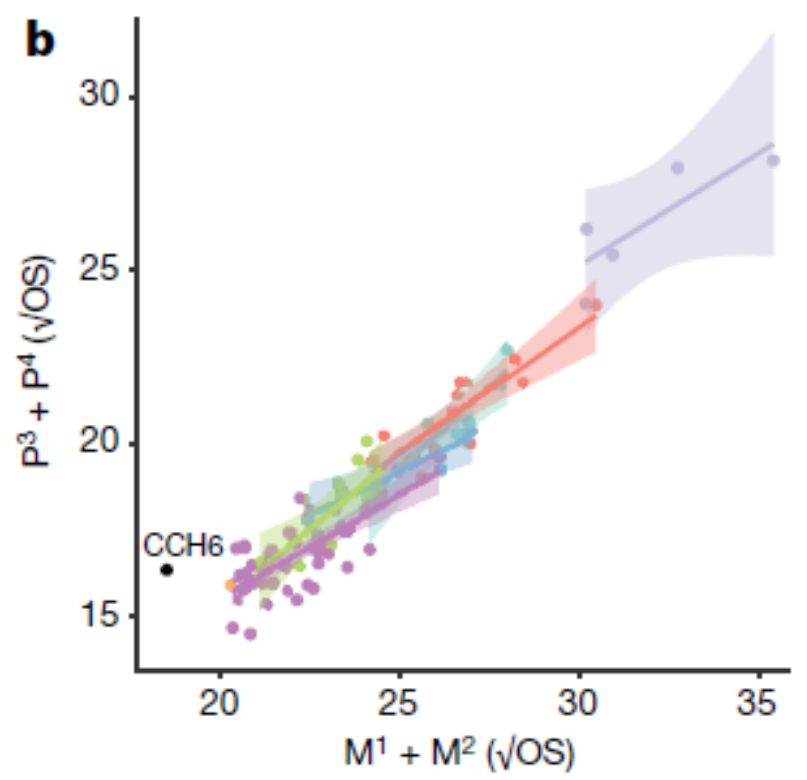

Fig. 3. Dental metrics. a, Square root of the computed occlusal surface area of P3 ( $n=166)$, P4 ( $n$ $=167), \mathrm{M} 1(n=216), \mathrm{M} 2(n=185)$ and M3 $(n=129)$. Dots correspond to the group average, vertical bars and shaded areas correspond to \pm 2 s.e.m. $\sqrt{ }$ OS, square root of the occlusal surface. $b$, Bivariate scatter plot of the summed square root of computed occlusal surface areas of premolars (P3 and P4) versus molars (M1 and M2), with regression lines (solid lines) and 95\% confidence intervals (shaded areas) for all groups, except $H$. floresiensis and $H$. luzonensis. Sample sizes for a, b, respectively: Australopithecus, $n=119,6$; Paranthropus, $n=111,5$; African and European early Homo, $n=114$, 13; Asian early Homo, $n=74,5 ; H$. neanderthalensis, $n=126,12 ; H$. sapiens, $n=307,47 ;$ H. floresiensis, $n=4,1 ; H$. luzonensis, $n=8 *, 1(* \mathrm{CCH} 8$ treated as P3 and P4 in a). A detailed list of specimens can be found in Supplementary Table 4. 

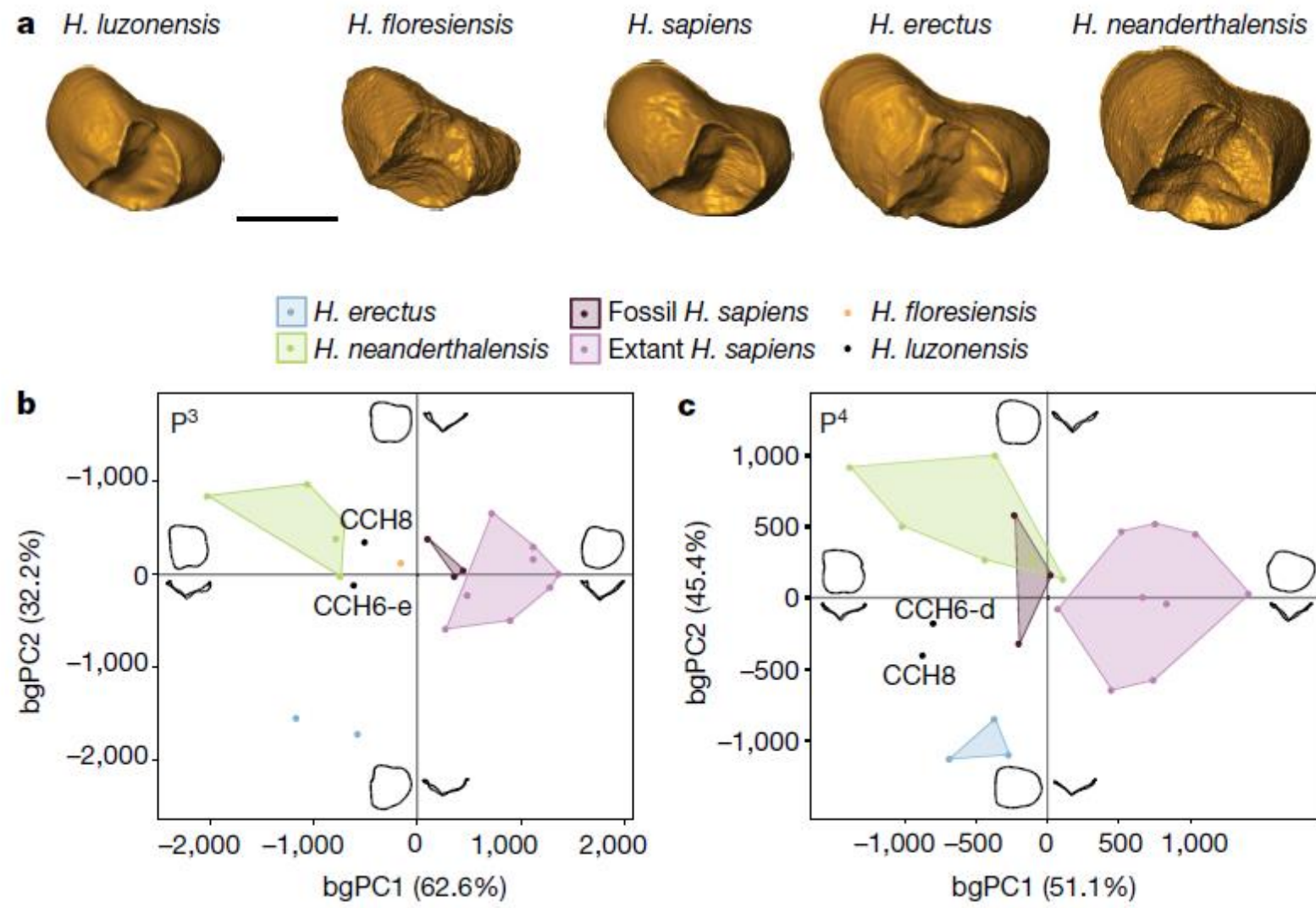

Fossil H. sapiens - H. floresiensis Extant $H$. sapiens • H. luzonensis

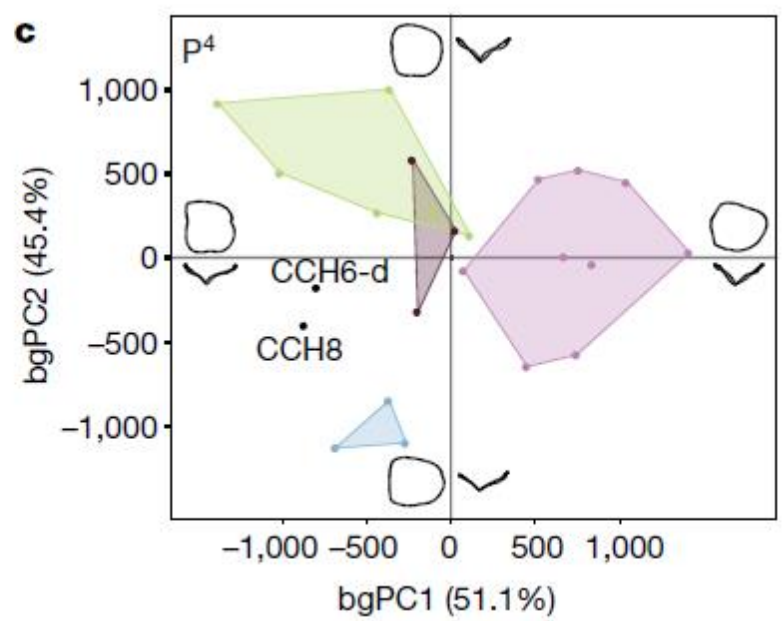

Fig. 4. Premolar EDJ of H. luzonensis. a, EDJ of the P3 of H. luzonensis (CCH6-e) compared to the EDJs of $H$. floresiensis (Liang Bua 1 (LB1)), H. sapiens, H. erectus (Sangiran 4) and $H$. neanderthalensis (KRD 53). Horns of dentine were reconstructed for CCH6-e, LB1 and Sangiran 4; see Methods. Scale bar, $5 \mathrm{~mm}$. b, c, Between-group principal component analyses (bgPCAs) of the three-dimensional landmark Procrustes registered shape coordinates of the P3s (b) and P4s (c). Sample sizes for b, c, respectively: H. erectus, $n=2,3 ; H$. neanderthalensis, $n=5$, 6 ; fossil $H$. sapiens, $n=3,3$; extant $H$. sapiens, $n=8,9 ; H$. floresiensis, $n=1,0 ; H$. luzonensis, $n=2,2$. A detailed list of specimens can be found in Supplementary Table 6. 


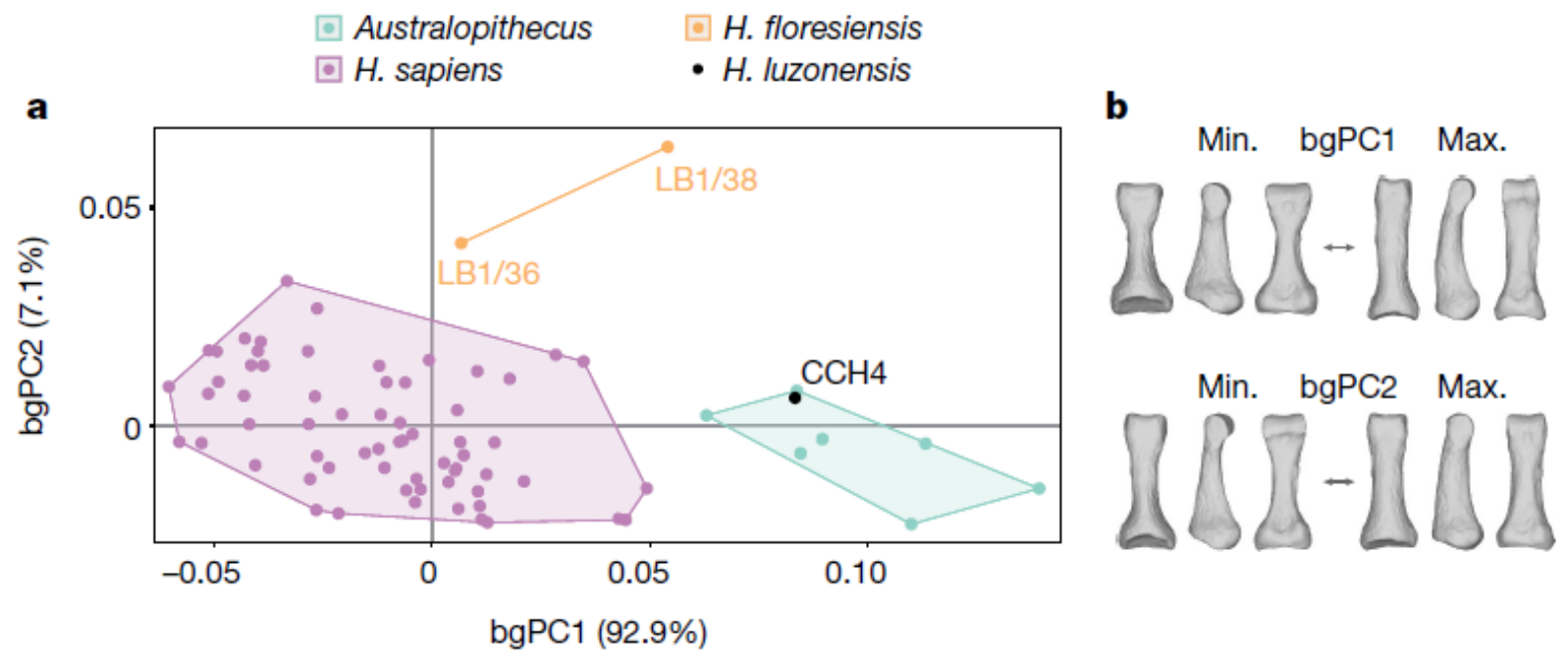

Fig. 5. Proximal pedal phalanx of $H$. luzonensis (CCH4). CCH4 compared with specimens attributed to Australopithecus (A. afarensis, $\mathrm{n}=6 ;$ A. africanus, $\mathrm{n}=1)$, recent $H$. sapiens $(\mathrm{n}=64)$ and $H$. floresiensis $(\mathrm{n}=2)$. a, bgPCA of Procrustes-registered landmarks and semilandmarks: scatter plot of individual scores for bgPC1 versus bgPC2. b, Shape variation associated with bgPC1 and bgPC2: CCH4 and Australopithecus phalanges are elongated and curved (see bgPC1 max.). A detailed list of specimens can be found in Supplementary Table 7. 


\section{Extended Data}

\begin{tabular}{|c|c|c|c|c|}
\hline Specienten No & Accession 140 & Dale of ascovery & Excswstion square & Idertifaton \\
\hline $\mathrm{CON1}$ & $11-77-\sqrt{3}-7691$ & 2007 May 5 & Square 1 & R thind mitatarair \\
\hline $\mathrm{cos}$ & $11-77 \sqrt{3}-10130$ & 2011 August 21 & Squere 1" & monus phalaru (ntermesiase) \\
\hline $\mathrm{COH} 3$ & $11-77-\sqrt{3}-10131$ & 2011 Angust 21 & Square $1^{L}$ & podal phalanx (hitermediaw) \\
\hline $\mathrm{COHA}$ & $11-77-\sqrt{3}-10132$ & 2011 Angus 21 & Square 1 & pedal (hatarox corcosimaf) \\
\hline cores & $11-77-\sqrt{3}-10133$ & 2011 Aaguet 21 & Square 1 & manali phatanx (dissai) \\
\hline $\mathrm{COH}-\mathrm{a}^{\mathrm{a}}$ & $11.77-\sqrt{3} \cdot 10136$ & 2011 August 24 & Square 1 & $R M^{3}$ \\
\hline $\mathrm{COH}-\mathrm{b}-\mathrm{a}=$ & $11-77-13-10137$ & 2011 August 24 & Square 1 & RM $M^{2}$ \\
\hline CON-ent & $11-77-\sqrt{3-1013 a}$ & 2011 Aupust 29 & Square 1 & $R M^{\prime}$ \\
\hline $\mathrm{CCH}^{2}-\mathrm{d}=\mathrm{T}$ & $11-77$ J3-10139 & 2011 August 24 & Square 1 & $R^{\prime \prime}$ \\
\hline COAB-atu & $11-77-\sqrt{3}-10140$ & 2011 Axquat 24 & Square 1 & R.p $p^{3}$ \\
\hline $\operatorname{con}$ & $\mid 1-77-\sqrt{3-1014 \mid}$ & 2011 Aaggust 20 & Square 5 & ferrur (sthat) \\
\hline $\mathrm{CCH}$ & $11-77$ J3-10216 & 2011 August 31 & Square 1 & L pir \\
\hline oOn & 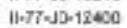 & S015 Juy 01 & Gquare 7 & $\mathrm{R} \mathrm{M}^{2}$ \\
\hline
\end{tabular}
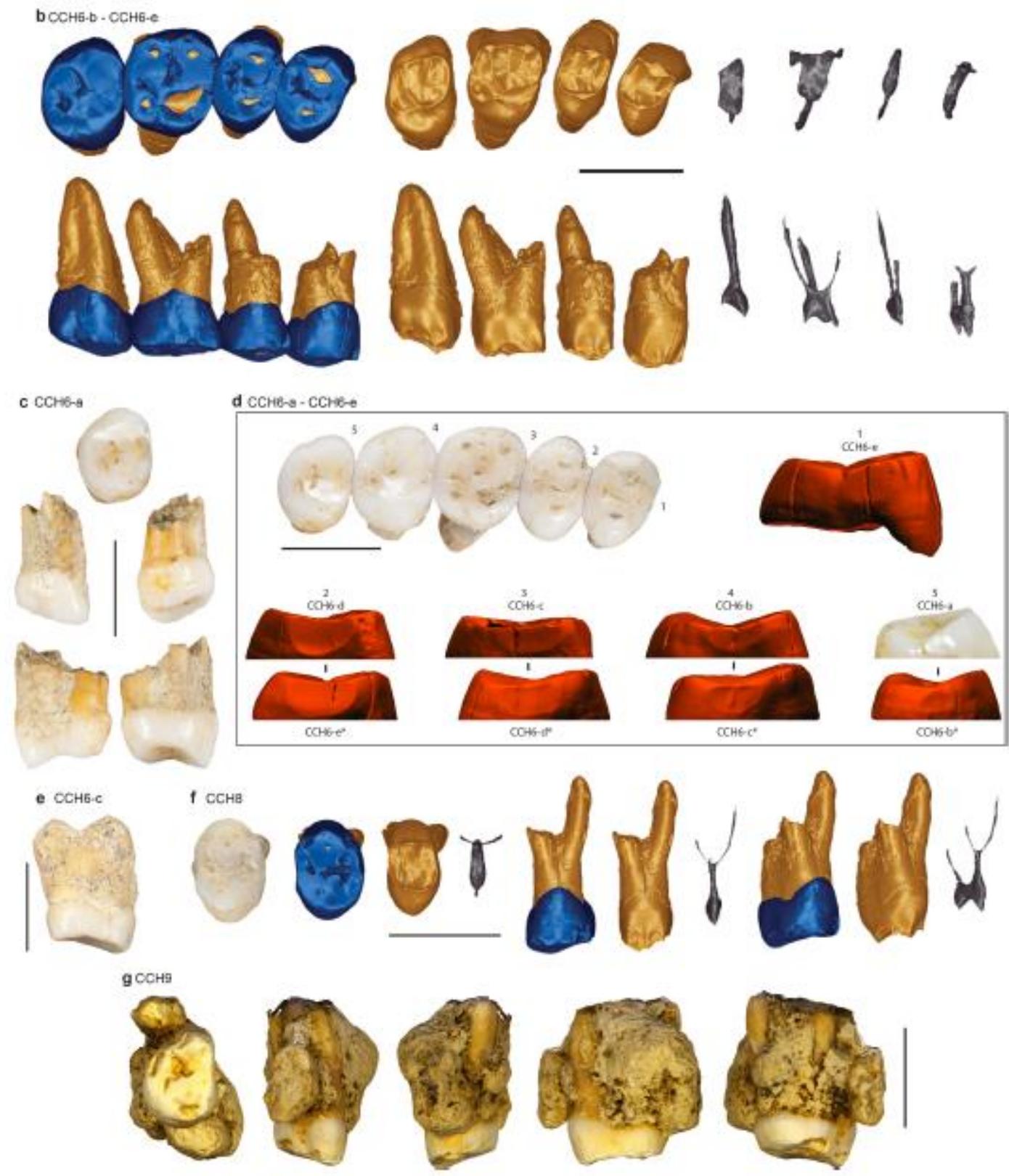
Extended Data Fig. 1. Inventory of the fossil elements attributed to $H$. luzonensis and detailed views of the dental remains. a, The hominin fossils recovered from Callao Cave. R, right; L, left; $\mathrm{P}$, premolar; M, molar. $\mathrm{b}$, Three-dimensional rendering of the postcanine maxillary teeth CCH6-b to CCH6-e (M2-P3): occlusal (top row) and buccal (bottom row) aspects. Enamel is shown in dark blue, dentine and cement in light brown and pulp cavity in dark grey. In all views, mesial is to the right, distal to the left. c, CCH6-a, right M3: occlusal, buccal, lingual, mesial and distal aspects (from top to bottom and left to right). Occlusal view: mesial is to the right, distal to the left. d, CCH6-a to CCH6-e, right M3-P3: photograph of occlusal aspect. Mesial is to the right, distal to the left. The numbers indicate the locations of the detailed views of the inter-proximal contact facets (IPCFs): P3 (CCH6-e), mesial IPCF 1: note the small size of this IPCF, indicating that the canine was probably not large; $2-5$ : note the perfect match between corresponding pairs of mesial (top row) and distal (bottom row, mirrored images) IPCFs, from the P3 (CCH6-e) to the M3 (CCH6-a). e, CCH6-c, right M1: distal aspect, showing the partially fused lingual and distobuccal roots. Lingual is to the left, buccal to the right. $\mathrm{f}, \mathrm{CCH} 8$, left P3 or P4, photograph of the original fossil (occlusal view) and three-dimensional rendering: occlusal (top row), buccal (middle row) and mesio-buccal (bottom row) aspects. Enamel is shown in dark blue, dentine and cement in light brown and pulp cavity in dark grey. g, CCH9, right M3: occlusal, buccal, lingual, mesial and distal aspects (from top to bottom and left to right). Occlusal view: mesial is to the right, distal to the left; captures of the three-dimensional surface model. Scale bars, $10 \mathrm{~mm}$ (IPCF views in $\mathrm{d}(1-5)$ are not to scale); the mirrored image is indicated by an asterisk. 

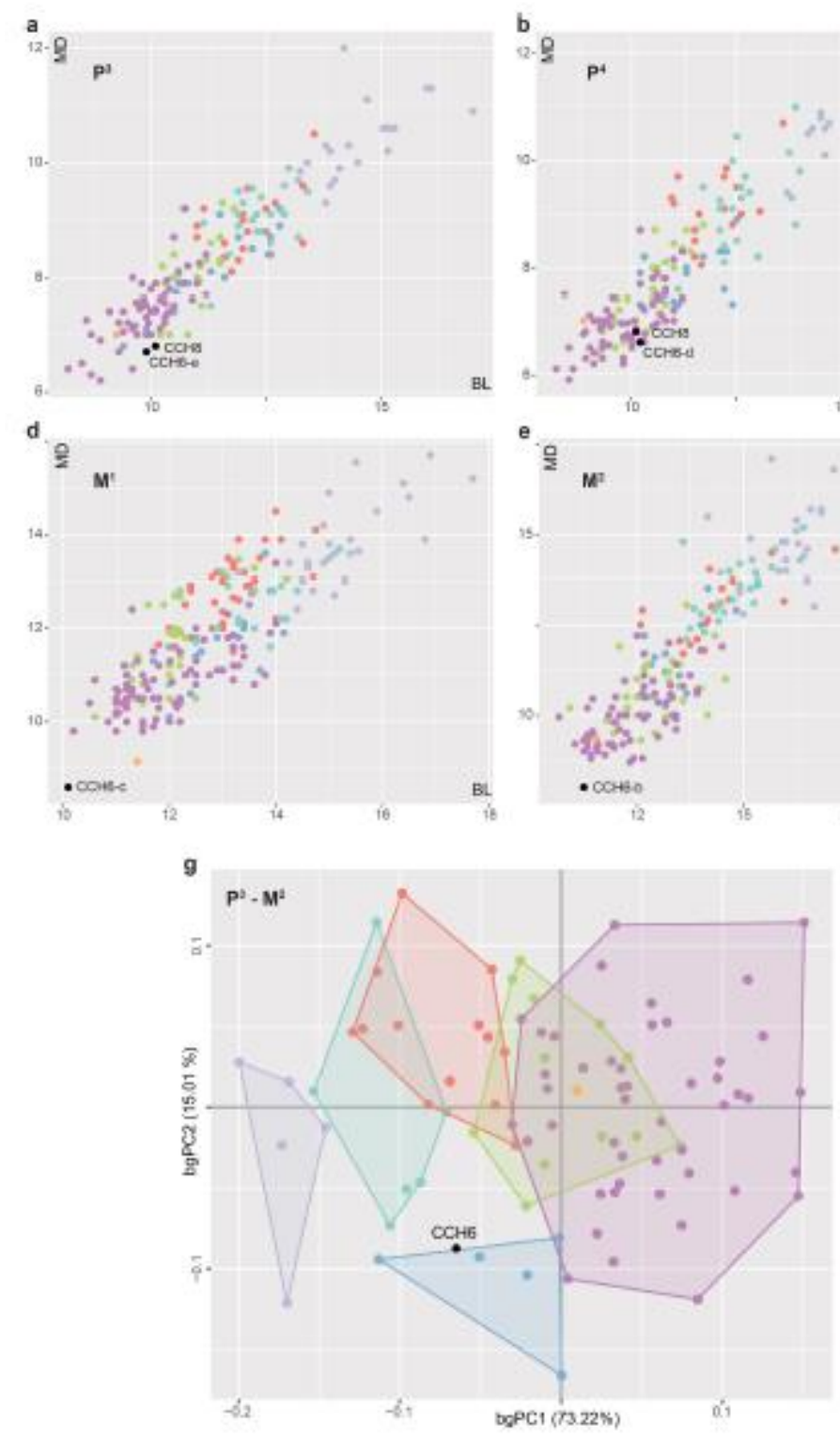

h
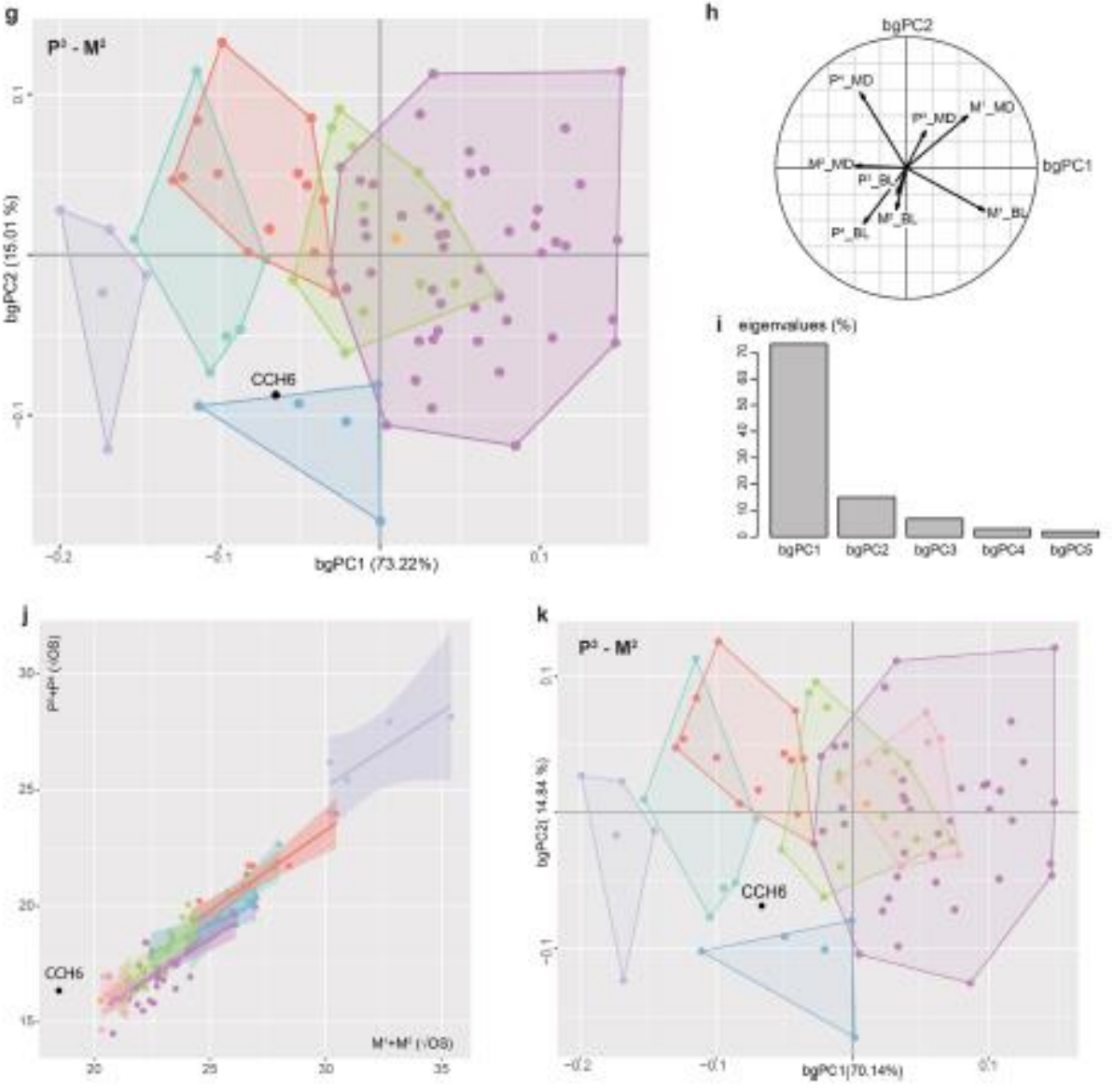

Extended Data Fig. 2. Dental metrics. a, b, d-f, Bivariate scatter plots for mesio-distal (MD) versus bucco-lingual (BL) diameters of P3 (a), P4 (b), M1 (d), M2 (e) and M3 (f). c, Key for a, b, d-g, j, k. Sample sizes for a, b, d, e, f, respectively: Australopithecus, $n=23,23,21,26,26$; 
Paranthropus, $n=22$, 20, 26, 23, 20; African and European early Homo, $n=23,19,37,21,17$; Asian early Homo, $n=16$, 17, 17, 13, 11; H. neanderthalensis, $n=23,26,27,30,20 ; \mathrm{H}$. sapiens, $n=57,61,86,70,33 ; \mathrm{H}$. floresiensis, $n=1,1,1,1,0 ; \mathrm{H}$. luzonensis, $n=2 *, 2 *, 1,1,2(* \mathrm{CCH} 8$ is a P3 or a P4). A detailed list of specimens can be found in Supplementary Table 4. g-i, bgPCA of the log-shape ratios of bucco-lingual and mesio-distal diameters of four postcanine maxillary teeth (P3, P4, M1 and M2), CCH6 was treated as a supplementary individual and was plotted a posteriori. g, Scatter plot of specimens for bgPC1 versus bgPC2, with convex hulls for all groups, except $H$. floresiensis and H. luzonensis. Sample sizes: Australopithecus, $n=6$; Paranthropus, $n$ = 5; African and European early Homo, $n=13$; Asian early Homo, $n=5$; H. neanderthalensis, $n$ $=12 ; \mathrm{H}$. sapiens, $n=47 ; \mathrm{H}$. floresiensis, $n=1 ; \mathrm{H}$. luzonensis, $n=1$. A detailed list of specimens can be found in Supplementary Table 4. h, Variable scores for bgPC1 versus bgPC2 (correlation circle; $\log$-shape ratios of variables). i, Bar plot of eigenvalues (\%) of bgPC1-bgPC5. j, k, Bivariate scatter plot of the summed square root of computed occlusal surface areas of premolars versus molars ( $\mathrm{j}$ ) and bgPCA of the log-shape ratios of buccolingual and mesio-distal diameters of four postcanine maxillary teeth (k) similar to the analyses presented in Fig. $3 \mathrm{~b}$ and in $\mathrm{g}$, respectively, but with 'Negritos' treated as a separate group (same sample sizes as in g, except for: $H$. sapiens, $n=38$ and $H$. sapiens 'Negritos', $n=9$ ). 

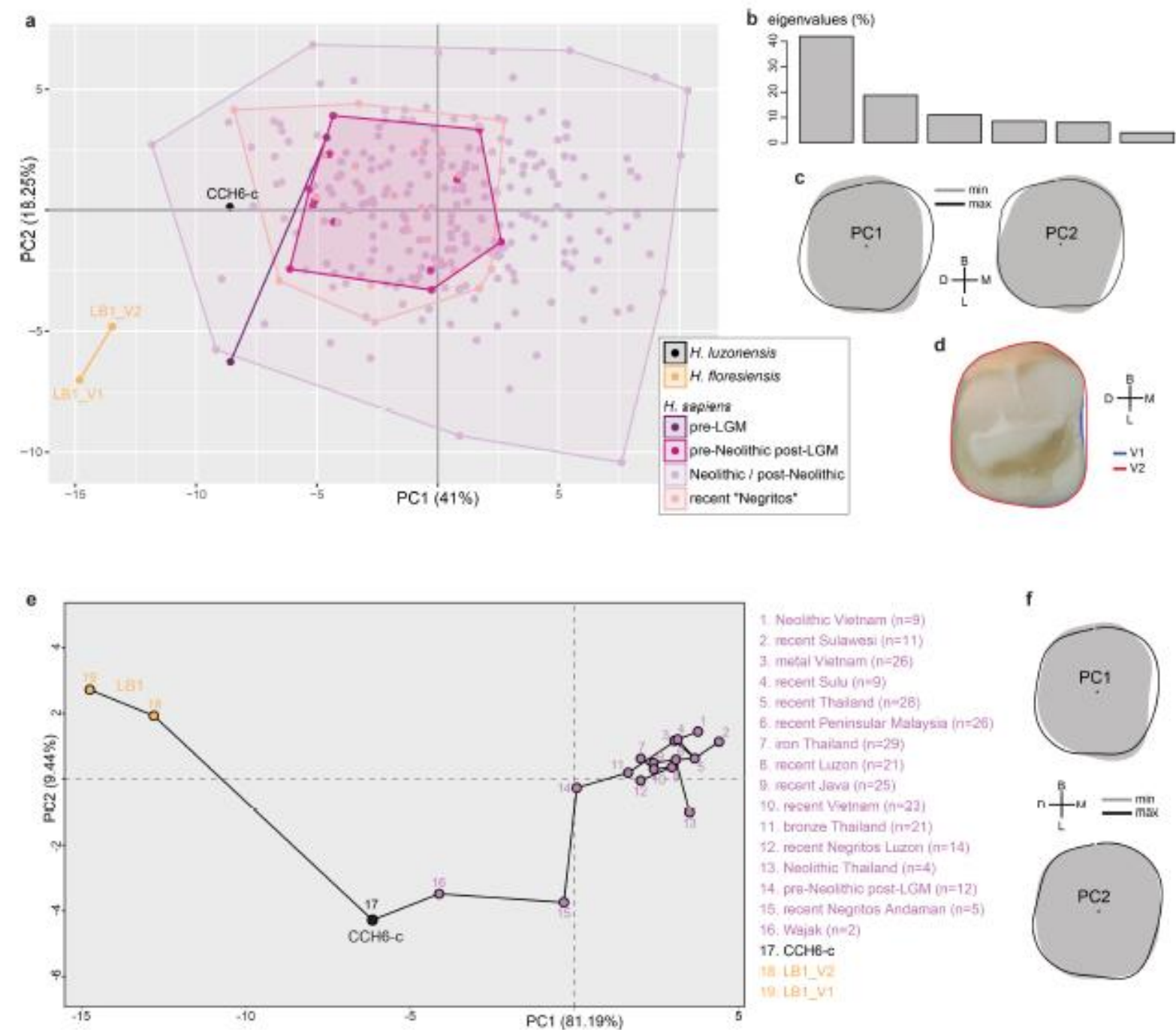

Extended Data Fig. 3. Elliptic Fourier analysis of M1 crown contour. CCH6-c compared to the holotype of $H$. floresiensis (LB1) and large samples of archaeological and recent $H$. sapiens individuals. a, PCA of shape data for all specimens, scatter plot of individual scores for PC1 versus PC2 (see Methods; elliptic Fourier descriptors applied to Procrustes-aligned outlines, ten harmonics included). LGM, Last Glacial Maximum. Sample sizes: $H$. luzonensis, $n=1 ; H$. floresiensis, $n=2$; pre-LGM, $n=2$; pre-Neolithic post-LGM, $n=12$; Neolithic/post-Neolithic, $n$ $=232$; recent 'Negritos', $n=19$. A detailed list of specimens can be found in Supplementary Table 5. b, Bar plot of eigenvalues (\%) of PC1-PC6. c, Extreme shape variations along PC1 and PC2. The scores of $H$. luzonensis $\mathrm{M} 1$ along PC1 and PC2 reflects a crown outline shape that is mesio-distally compressed, but not as much as that of $H$. floresiensis (two versions of the LB1 right M1). d, Right M1 of the holotype of $H$. floresiensis LB1 showing the two different versions of the crown outline (see Methods): the original contour (V1; in blue) published in a previous study7, and the contour (V2; in red) drawn by J.C. differ in the compensation of the mesial IPCF. These two versions differ minimally in the results of the elliptic Fourier analysis (see $d$ and $h$ ). e, PCA of Fourier descriptors for the means of 16 groups of $H$. sapiens (sample sizes in brackets, see details in Supplementary Table 5), H. luzonensis CCH6-c and H. floresiensis LB1 (V1 and V2 treated as 2 groups): scatter plot of mean scores for PC1 versus PC2 with a superimposed 
minimum spanning tree indicating distances between groups. f, Extreme shape variations along PC1 and PC2: H. floresiensis differs from H. luzonensis in having a M1 crown contour shape that is more compressed mesio-distally, with a more developed protocone. 

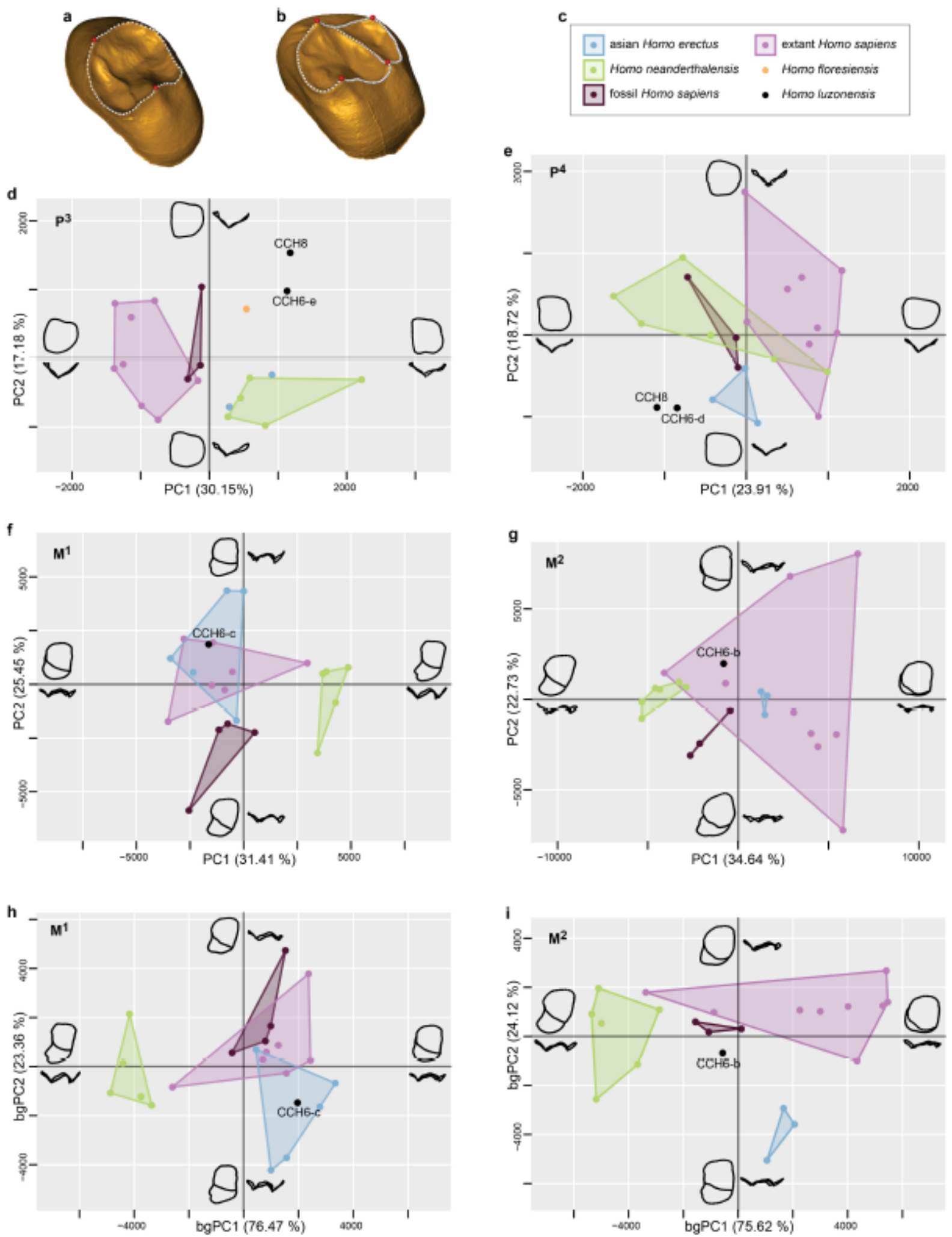

Extended Data Fig. 4. Procrustes analyses of the premolar and molar EDJ. a, Landmarks placed on the main dentine horns (in red) and semilandmarks (in white) positioned along the marginal ridges of the premolar EDJ. b, Landmarks placed on the main dentine horns (in red) and the 
semilandmarks (in white) positioned along the marginal ridges and on the oblique crest of the molar EDJ. c, Key for d-i. d-g, PCAs of the three-dimensional landmarks Procrustes-registered shape coordinates of the P3s (d), P4s (e), M1s (f) and M2s (g). h, i, bgPCAs of the three dimensional landmarks Procrustes-registered shape coordinates of the M1s (h) and M2s (i). Sample sizes for $\mathrm{d}$, e, f and $\mathrm{h}$, and $\mathrm{g}$ and i, respectively: $H$. erectus, $n=2,3,5$, and $3 ; H$. neanderthalensis, $n=5,6,5$, and 6; fossil $H$. sapiens, $n=3,3$, 4, and 3; extant $H$. sapiens, $n=8$, 9, 7, and 9; H. floresiensis, $n=1,0,0$, and 0; H. luzonensis, $n=2,2,1$, and 1 . A detailed list of specimens can be found in Supplementary Table 6. 

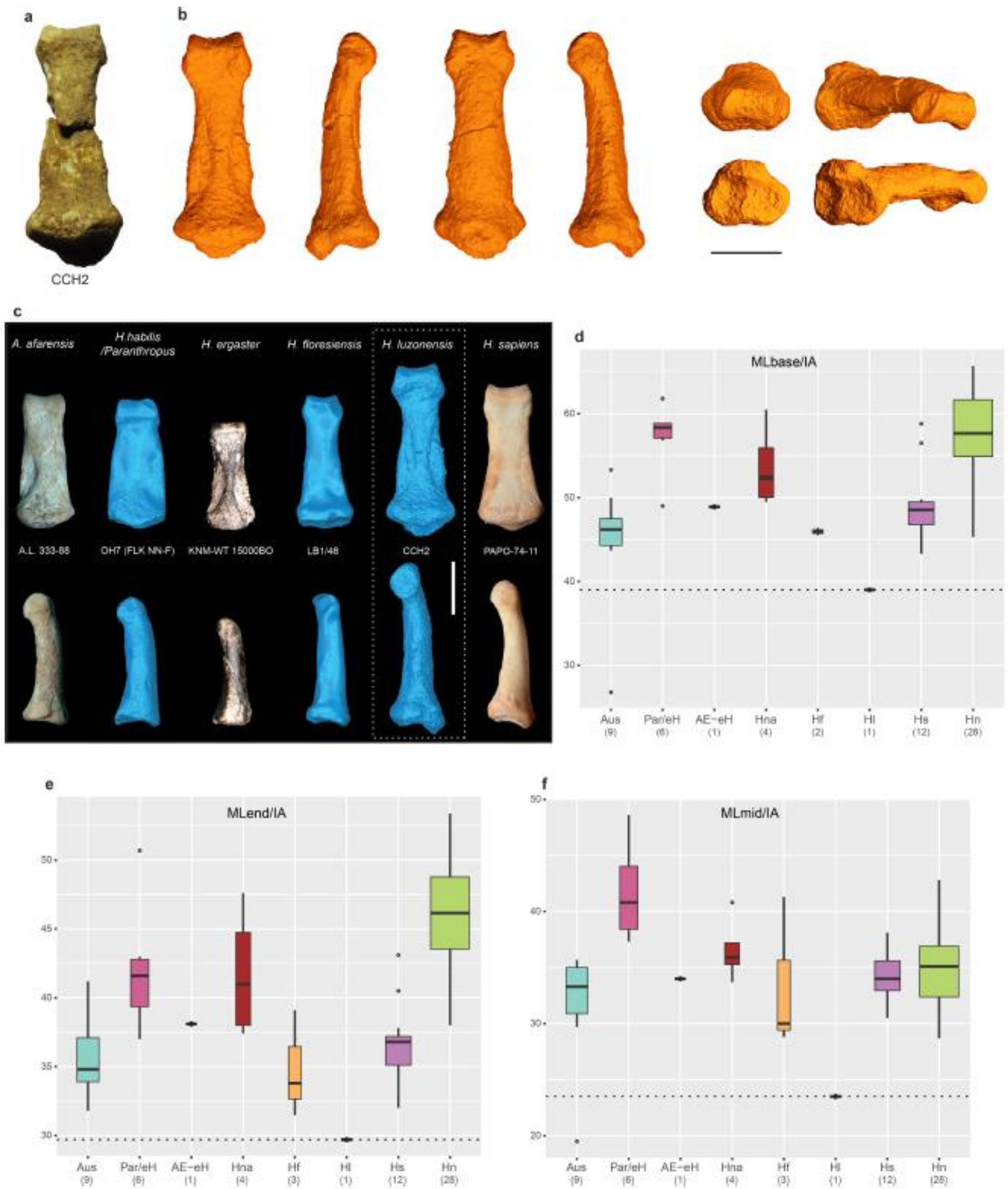

Extended Data Fig. 5. CCH2, intermediate manual phalanx of $H$. luzonensis. a, Photograph of the original specimen $\mathrm{CCH} 2$ in palmar view. b, Three-dimensional rendering of $\mathrm{CCH} 2$. From left to right: palmar, lateral, dorsal, medial, distal (top), proximal (bottom), disto-lateral and proximolateral aspects. c, Comparison of $\mathrm{CCH} 2$ with Pliocene (A.L.333-88), Lower Pleistocene (OH7 (FLK NN-F) and KNM-WT 15000BO), Upper Pleistocene (LB1/48) and recent (PAPO-74-11) intermediate manual phalanges in palmar (top) and side (bottom) views. All specimens are from 
rays 2-5 of unknown side, except for $\mathrm{OH} 7$ (third ray, probably from the right hand of a juvenile individual). Note the unique proximally accentuated beak located on the dorsum of the proximal surface of $\mathrm{CCH} 2$. d-f, Relative robusticity of the intermediate manual phalanx $\mathrm{CCH} 2$. Box-andwhisker plots depicting the ratio of the interarticular length (IA)

and the maximum medio-lateral width of the base (MLbase) (d), the head (MLend) (e) and the midshaft (MLmid) (f). Box, 25-75th percentiles; centre line, median; whiskers, non-outlier range; dots, outliers; dotted line, value for $\mathrm{CCH} 2$. AE-eH, African and European early Homo; Aus, Australopithecus; Hf, H. floresiensis; Hl, H. luzonensis; Hna, H. naledi; Hn, H. neanderthalensis; Hs, H. sapiens, Par/eH, Paranthropus/early Homo. $n$ indicates sample size. A detailed list of specimens can be found in Supplementary Table 8. For conservative reasons, taxonomic assignation of hand remains of $\mathrm{OH} 7$ and several SKX specimens are considered to be uncertain (see main text and Methods). Scale bars, $10 \mathrm{~mm}$. 

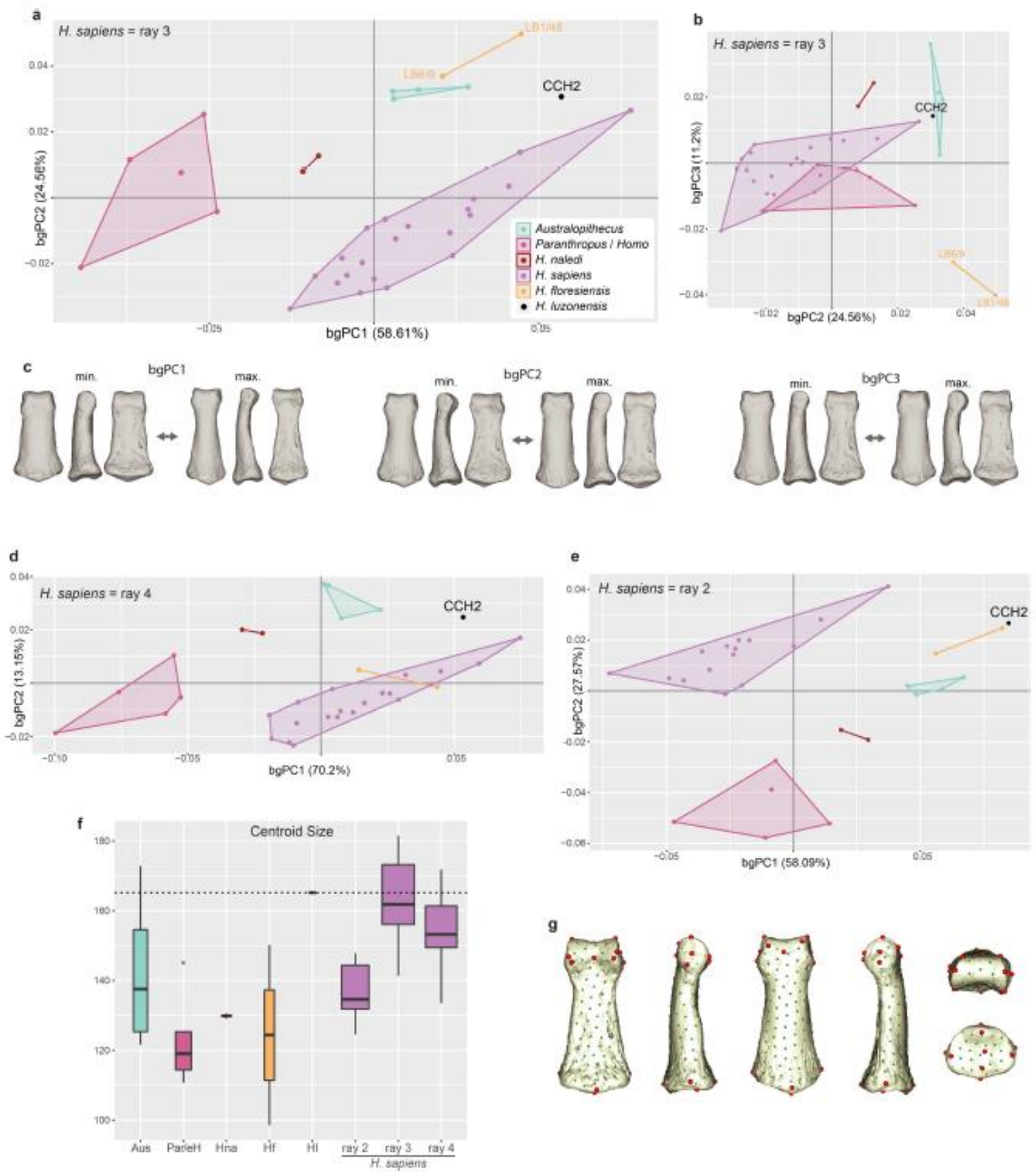

Extended Data Fig. 7. CCH5, distal manual phalanx of H. luzonensis. a, Photograph of the original specimen $\mathrm{CCH} 5$ in palmar view. b, Three dimensional rendering of $\mathrm{CCH}$. From left to right: palmar, lateral/medial, dorsal, medial/lateral, distal (top), proximal (bottom), disto-lateral/medial and proximolateral/medial aspects. c, Comparison of CCH5 with Pliocene (A.L.333-11 and A.L.333-50), Lower Pleistocene (OH7 (FLKNN-B) and SKX 27504), Upper Pleistocene (LB6/12) and recent distal manual phalanges (PAPO-74-53 and PAPO-74-11) in palmar (top) and side (bottom) views. All specimens are from rays 2-5 of unknown side, except for $\mathrm{OH} 7$ (second to fourth ray, probably from the right hand of a juvenile individual). $d, e$, Box-and-whisker plots depicting the expansion index ((apical tuft maximum medio-lateral width/maximum mediolateral width of the base $) \times 100)$ ) $(\mathrm{d})$ and the robusticity index $(($ apical tuft maximum medio-lateral width/biomechanical length $) \times 100)(e)$ of the distal manual phalanx CCH5. Box, 25-75th percentiles, centre line, median; whiskers, non-outlier range; dots, outliers; dotted 
line, value for CCH5. A detailed list of specimens can be found in Supplementary Table 10. For conservative reasons, taxonomic assignation of hand remains OH7 and SKX 27504 are considered to be uncertain (see main text and Methods). Scale bars, $10 \mathrm{~mm}$. 

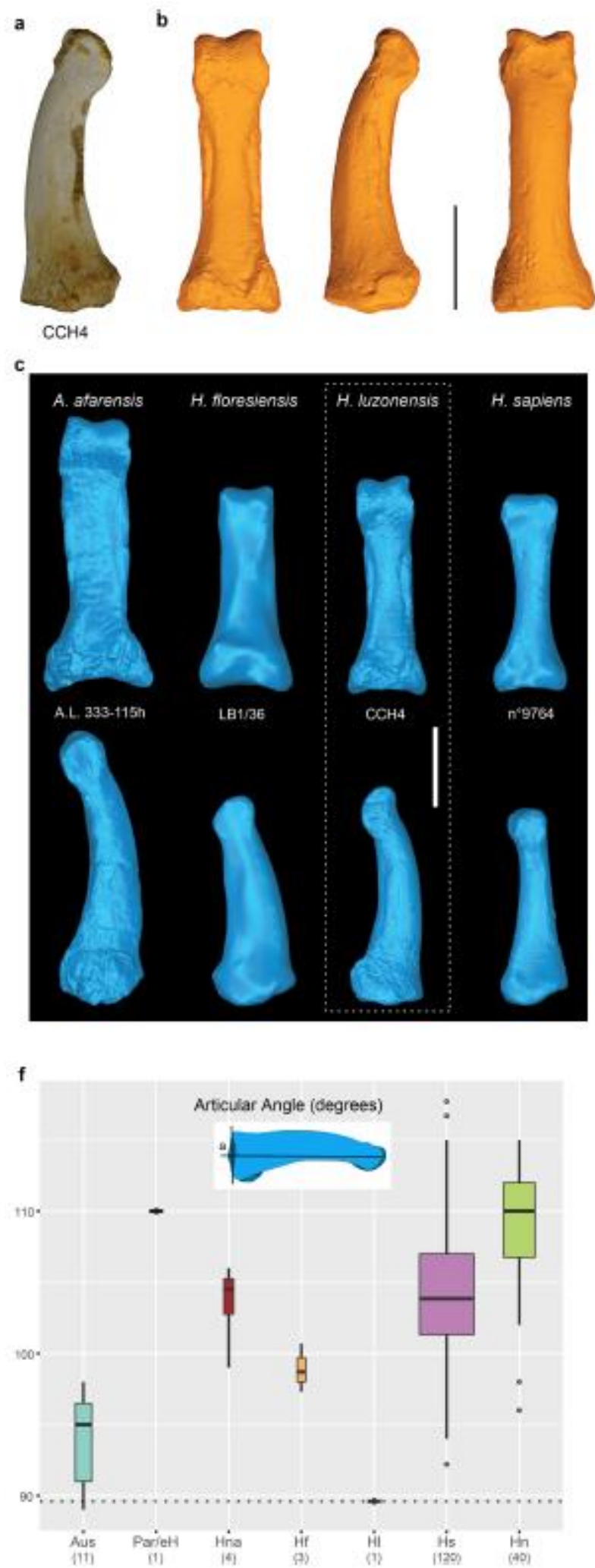
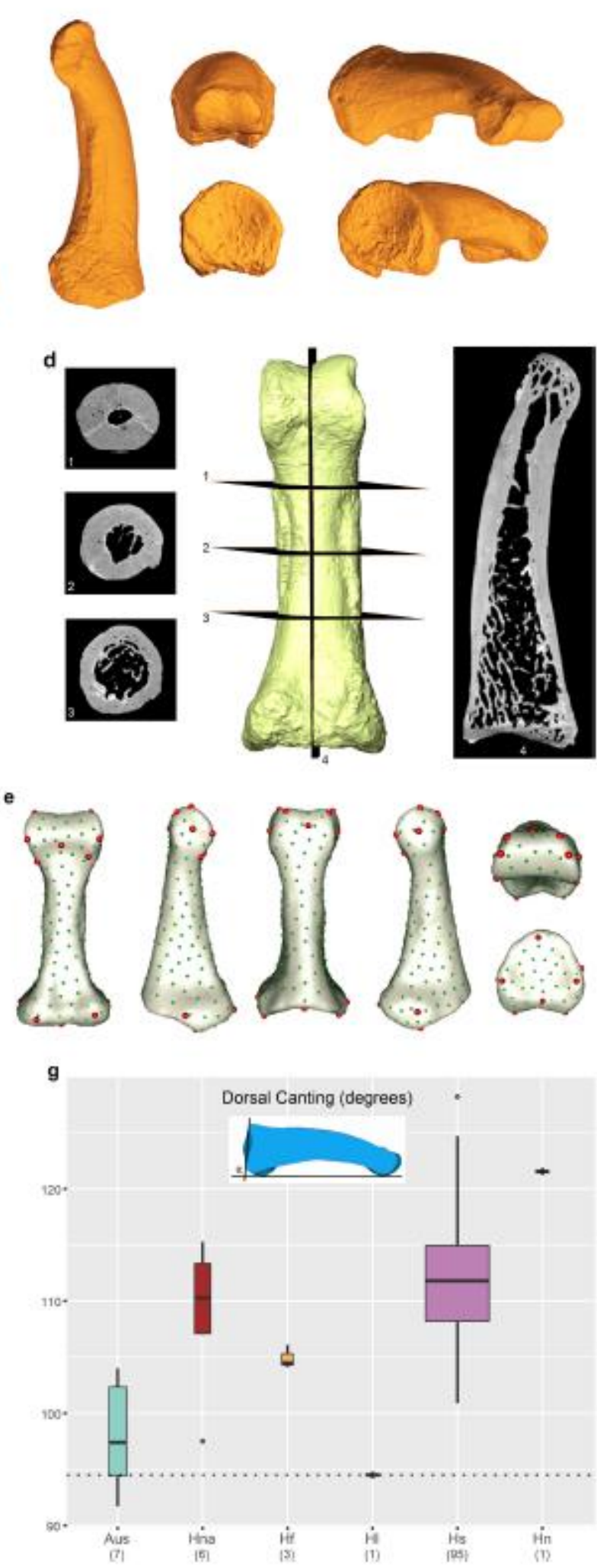

Extended Data Fig. 8. CCH4, proximal pedal phalanx of H. luzonensis. a, Photograph of the original specimen $\mathrm{CCH} 4$ in lateral view. b, Three dimensional rendering of $\mathrm{CCH} 4$. From left to right: plantar, lateral, dorsal, medial, distal (top), proximal (bottom), disto-lateral and proximo- 
lateral aspects. c, Comparison of CCH4 with Pliocene (A.L.333-115h, left third proximal phalanx, mirrored), Pleistocene (LB1/36, unknown side and rays 2-5) and recent ('Negrito' 9764, right third proximal pedal phalanx), proximal pedal phalanges in plantar (top) and side (bottom) views. d, Transverse $(1,2,3)$ and mid-sagittal (4) micro-CT slices of CCH4 (plantar aspect of three-dimensional rendering, during the segmentation process; $1,2,3$, dorsal is up, plantar is down, lateral is left, medial is right; 4, distal is up, proximal is down, dorsal is left, plantar is right). e, Protocol for three-dimensional Procrustes analysis: landmarks placed on the main anatomical features $(n=20$, in red) and equally spaced semilandmarks $(n=250$, in green $)$ placed on the whole surface of the proximal pedal phalanx (template shown on the proximal pedal phalanx, ray 2 of the right foot of the recent $H$. sapiens 35071). f, g, Box-and-whisker plots depicting comparisons of the articular angle $a$ (f) and the dorsal canting angle $\alpha(\mathrm{g})$ of the proximal pedal phalanx CCH4. Box, 25-75th percentiles; centre line, median; whiskers, nonoutlier range; dots, outliers; dotted line, value for $\mathrm{CCH} 4$. A detailed list of specimens can be found in Supplementary Table 11. Scale bars, $10 \mathrm{~mm}$. 
a

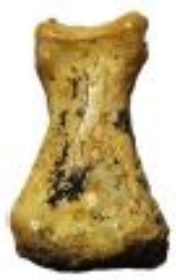

$\mathrm{CCH}_{3}$ b

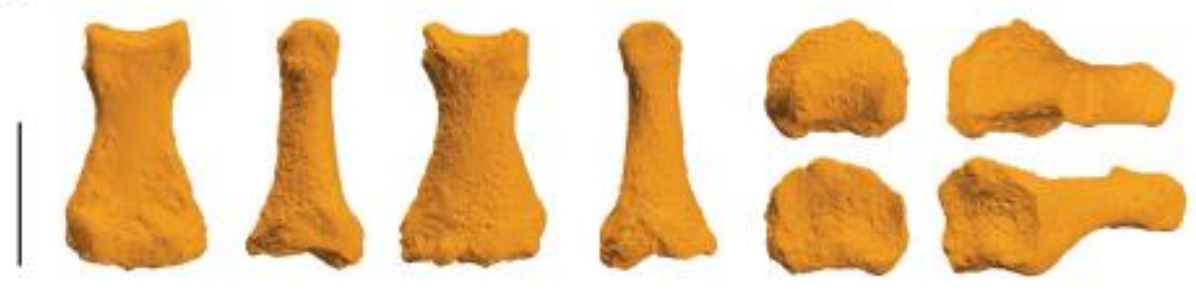

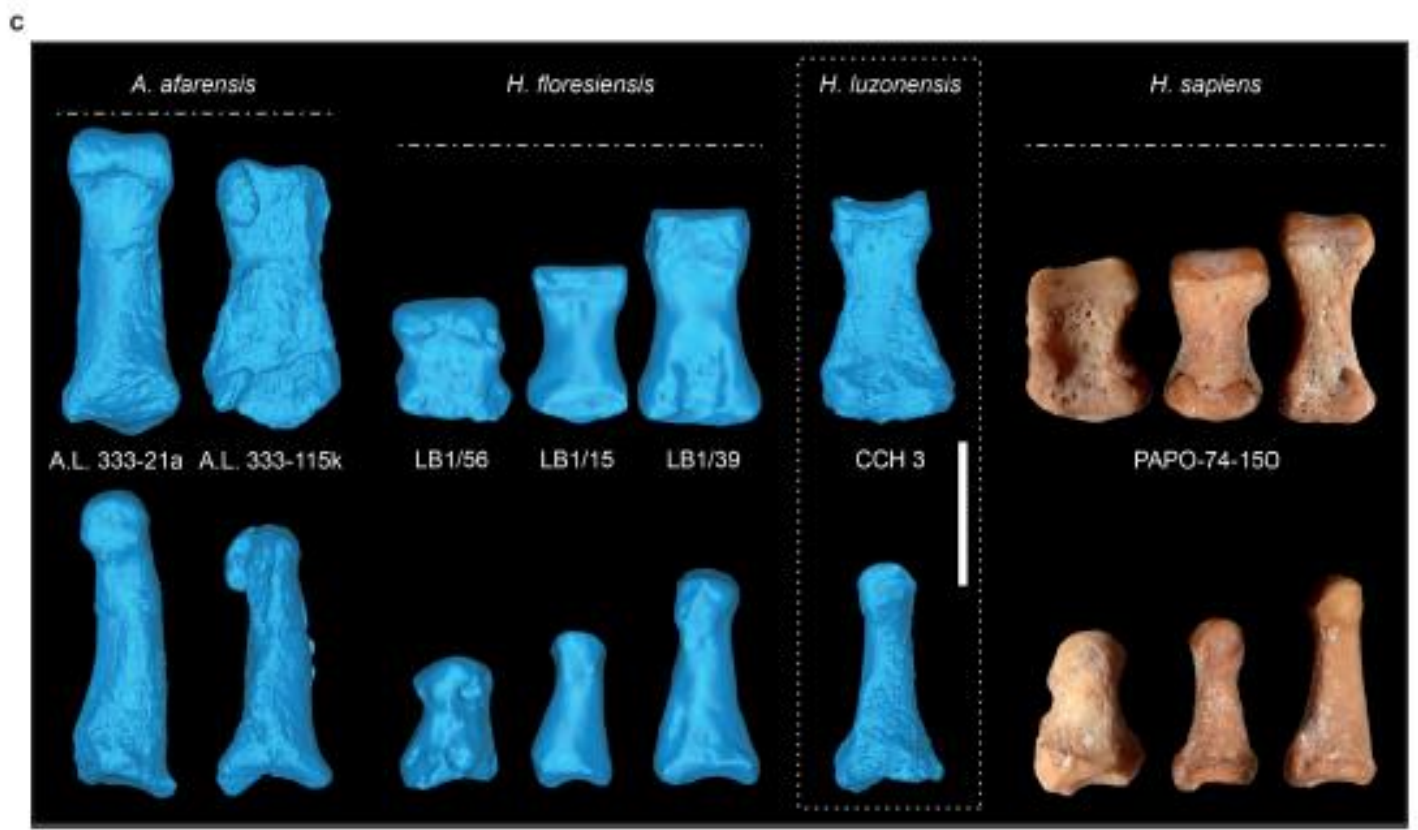

Extended Data Fig. 9. CCH3, intermediate pedal phalanx of $H$. luzonensis. a, Photograph of the original specimen $\mathrm{CCH} 3$ in plantar view. $b$, Three-dimensional rendering of $\mathrm{CCH} 3$. From left to right: plantar, medial, dorsal, lateral, distal (top), proximal (bottom), disto-medial and proximomedial aspects. c, Comparison of CCH3 with Pliocene (A.L.333-21a, unknown side and rays 2-5, and A.L.333-115k, fourth intermediate phalanx), Upper Pleistocene (LB1/56, LB1-15 and LB1/39: unknown side and rays 2-5) and recent (PAPO-74-150) intermediate pedal phalanges in plantar (top) and side (bottom) views. Note the variation in shape and size both between taxa (for example, $H$. sapiens and $H$. floresiensis) and in the same individual (for example, LB1 and PAPO-74-150). A detailed list of specimens can be found in Supplementary Table 12. Scale bars, $10 \mathrm{~mm}$. 

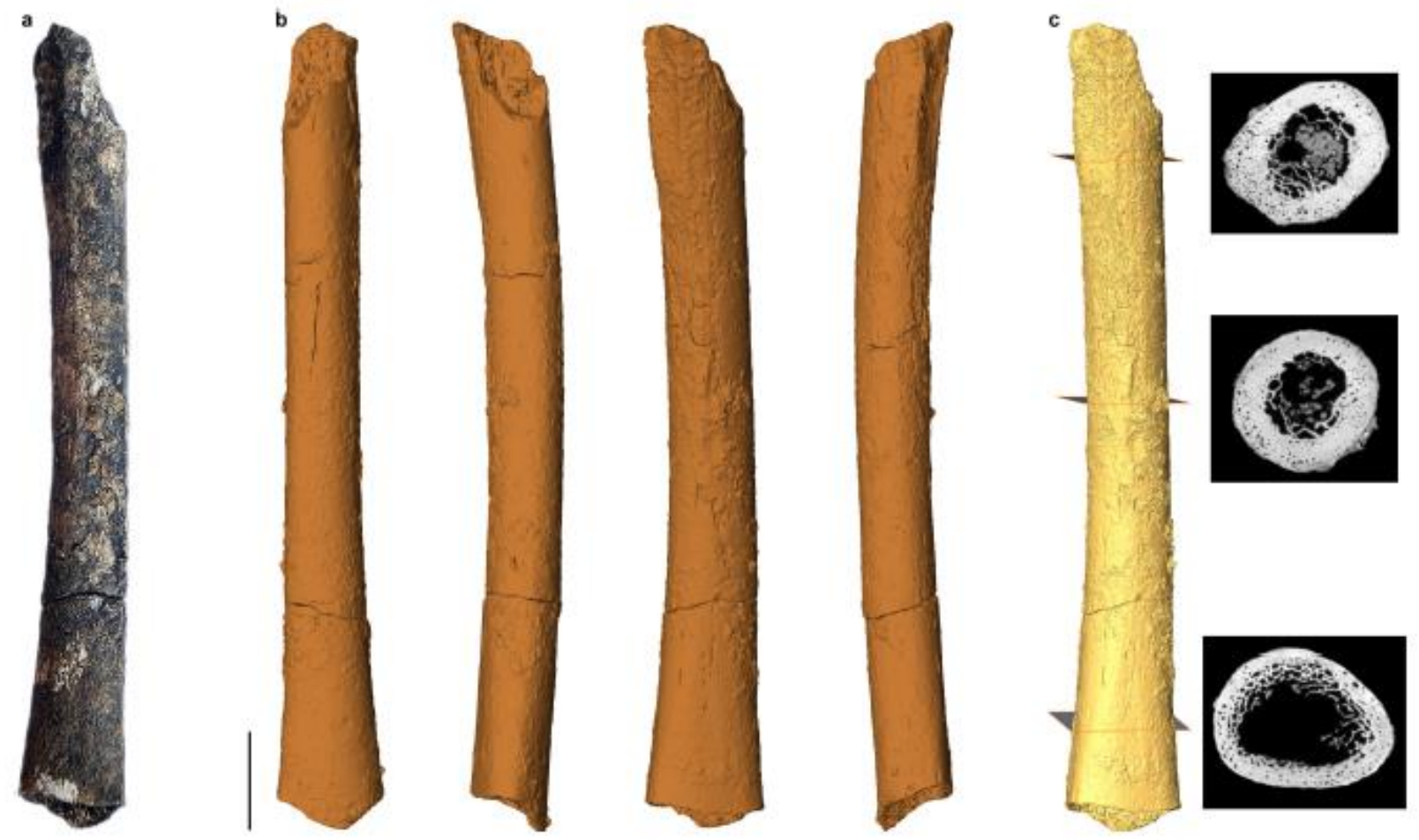

Extended Data Fig. 10. CCH7, femoral shaft of a juvenile individual of $H$. luzonensis. a, Photograph of the original specimen $\mathrm{CCH} 7$ (posterior aspect). b, Three-dimensional rendering of CCH7. From left to right: anterior, medial, posterior and lateral aspects. Scale bar, $20 \mathrm{~mm}$. c, Transverse micro-CT slices of $\mathrm{CCH} 7$ at proximal diaphysis (top), midshaft (middle) and distal diaphysis (bottom), and posterior aspect of the three-dimensional rendering of the femoral shaft, during the segmentation process (orientation of slices: anterior is up, posterior is down, lateral is left, medial is right). 